\title{
THEORETICAL AND NUMERICAL STUDY OF A FREE BOUNDARY PROBLEM BY BOUNDARY INTEGRAL METHODS
}

\author{
Michel Crouzeix ${ }^{1}$, Philippe FÉat ${ }^{1}$ and Francisco-Javier Sayas ${ }^{2}$
}

\begin{abstract}
In this paper we study a free boundary problem appearing in electromagnetism and its numerical approximation by means of boundary integral methods. Once the problem is written in a equivalent integro-differential form, with the arc parametrization of the boundary as unknown, we analyse it in this new setting. Then we consider Galerkin and collocation methods with trigonometric polynomial and spline curves as approximate solutions.

Résumé. Dans cet article on considère un problème à frontière libre intervenant en formage électromagnétique. Après l'avoir ramené à un système intégro-différentiel où l'inconnue est la représentation paramétrique de la frontière, on en étudie les propriétés mathématiques essentielles. On s'intéresse ensuite à l'approximation numérique par des méthodes de type Galerkin ou de collocation en utilisant pour l'approximation des polynômes trigonométriques ou des fonctions splines.
\end{abstract}

Mathematics Subject Classification. 35R35, 41A15, 42A12, 45G05, 65R20.

Received: November 30, 2000. Revised: July 7, 2001.

\section{INTRODUCTION}

This paper is concerned with the overdetermined boundary value problem

$$
\begin{cases}-\Delta u=j, & \text { in } \Omega, \\ u=0, & \text { in } \mathbb{R}^{2} \backslash \bar{\Omega} \\ \partial_{n} u=-\lambda, & \text { on } \partial \Omega,\end{cases}
$$

where $j$ is a function whose support lies on a compact set strictly contained in the bounded open set $\Omega, \lambda$ is a real number and $u \in \mathrm{H}^{1}\left(\mathbb{R}^{2}\right)$. If, for instance, $j$ and $\Omega$ are known, the two first equations determine $u$. Then, the third one gives an overdetermination which can be used to find $\Omega$.

Some results about existence, uniqueness for this kind of free boundary problem, as well as regularity properties of the boundary can be found in [1]. Among the big amount of literature which has followed this deep paper we refer to $[3,4,6,7]$ and $[9]$ for complementary facts; the last paper is devoted to the inverse problem, $\Omega$ given find $j$.

Keywords and phrases. Free boundary, spline, trigonometric polynomial.

1 Institut de Recherche Mathématique de Rennes, UMR CNRS 6625, Université de Rennes 1, Campus de Beaulieu, Rennes, France. e-mail: Michel.Crouzeix@univ.rennes1.fr

2 Dep. Matemática Aplicada, Universidad de Zaragoza, Centro Politécnico Superior, c/ María de Luna, 3-50015 Zaragoza, Spain. 
The problem is often stated in a variational context. For instance in [13], given $j$ and a real constant $\gamma$, it is looking for $u \in \mathrm{H}^{1}(\Omega)$ minimising the functional

$$
\frac{1}{2} \int|\nabla v|^{2} \mathrm{~d} x-\int j v \mathrm{~d} x
$$

with the constraint that the measure of the support of $u$ is $\gamma$. The parameter $\lambda$ appears then as a Lagrange multiplier. It is possible to develop numerical methods based on such a variational setting [14] but the convergence analysis seems not an easy task.

Our approach hinges on considering the arc parameterization of the boundary as the unknown of the problem. A similar approach can be found in [13] using polar coordinates. An alternative possibility is developed in [2]; they approximate the conformal map which apply the exterior of the unit disk onto the exterior of $\Omega$.

In Section 2 we write the free boundary problem in the equivalent integral form. We state some properties of the solution as well as recall the functional frame in Sections 3 and 4 . Four families of numerical methods for the problem are introduced and analysed in this paper, namely, Galerkin and collocation methods with trigonometric polynomials and smoothest splines as trial functions. These methods are introduced in Section 5 , where we give the general analysis of projection methods for the problem, under three hypotheses which will have to be verified for each of the methods. To simplify the presentation, the analysis of this section is carried out avoiding the study of dependence on the parameter $\lambda$. Section 6 solves this question on each regular branch of the solution.

The final sections of this work are then devoted to verification of the hypotheses given in previous sections. We then derive convergence estimates for each of the methods. This task is fully achieved for trigonometric methods in Sections 7, 8 and 9. For the spline Galerkin method (Sect. 10), we give a sufficient condition for the hypotheses to hold. This condition is uniquely related to the sequence of grids and to the $\mathrm{L}^{2}$ orthogonal projection onto spline spaces and holds, for instance, for quasi-uniform sequences of grids. The study of more general situations where this condition is satisfied is an open question but, nevertheless, completely unrelated to our problem. A similar program is then developed for the collocation method in Section 11. A short description of numerical experiments is given in the last section.

\section{Statement of the PRoblem}

Let $\lambda$ be a real number and $j$ be a compactly supported function, which for the sake of simplicity we assume to belong to $\mathrm{L}^{\infty}\left(\mathbb{R}^{2}\right)$. This paper is concerned with the numerical solution of the following free boundary problem: find an open set $\Omega \subset \mathbb{R}^{2}$ and $u \in \mathrm{H}^{1}\left(\mathbb{R}^{2}\right)$ such that

$$
\begin{cases}-\Delta u=j, & \text { in } \Omega, \\ u=0, & \text { in } \mathbb{R}^{2} \backslash \bar{\Omega} \\ \partial_{n} u=-\lambda, & \text { on } \partial \Omega .\end{cases}
$$

We do not consider the case $\lambda=0$ (or equivalently $\int_{\mathbb{R}^{2}} j(y) \mathrm{d} y=0$ ) which corresponds to a too singular situation (see $[3])$. By a change of scale in $u$ ( $\Omega$ remains unchanged) we can therefore demand

$$
\int_{\mathbb{R}^{2}} j(y) \mathrm{d} y=2 \pi
$$

and by a translation of the origin in $\mathbb{R}^{2}$, that

$$
\int_{\mathbb{R}^{2}} y_{k} j(y) \mathrm{d} y=0, \quad k=1,2
$$

Let $K:=\operatorname{supp} j$. 
If $K \subset \Omega$ and $\Gamma:=\partial \Omega$ is a Lipschitz boundary then (1) is equivalent to

$$
\left\{\begin{array}{l}
\lambda \text { length } \Gamma=2 \pi, \\
\frac{\lambda}{2 \pi} \int_{\Gamma} \log |\cdot-y| \mathrm{d} \sigma_{y}-\frac{1}{2 \pi} \int_{K} j(y) \log |\cdot-y| \mathrm{d} y=0, \quad \text { on } \Gamma,
\end{array}\right.
$$

by means of the boundary representation

$$
u(\cdot)=\frac{\lambda}{2 \pi} \int_{\Gamma} \log |\cdot-y| \mathrm{d} \sigma_{y}-\frac{1}{2 \pi} \int_{K} j(y) \log |\cdot-y| \mathrm{d} y, \quad \text { in } \mathbb{R}^{2} .
$$

Indeed it is clear that (1) implies (4). For the converse part we define a continuous function $u$ by (5). Then we have $-\Delta u=j$ in $\mathbb{R}^{2} \backslash \Gamma$ and $u=0$ on $\Gamma$. From (2) we see that the function $u$ satisfies $u(x) \rightarrow 0$ as $x \rightarrow \infty$. Together with $u=0$ on $\Gamma$ and the harmonicity of $u$ outside $\Omega$, we deduce from the maximum principle that $u=0$ outside $\Omega$. Using integration by parts we obtain from (4) that $\int_{\Gamma}\left(\partial_{n} u+\lambda\right) \log |x-y| \mathrm{d} \sigma_{y}=0$, on $\Gamma$. We have also $\int_{\Gamma}\left(\partial_{n} u+\lambda\right) \mathrm{d} \sigma_{y}=0$, therefore $\partial_{n} u+\lambda=0$ on $\Gamma$.

In [5] it is proven that if $\Gamma$ is a $\mathrm{C}^{1}$ curve, then it is analytic (see also [1,9] for the same kind of result with a slightly different assumption). We will restrict henceforth to this case.

We introduce now a set of complex valued $2 \pi$-periodic functions

$$
E:=\left\{x \in \mathrm{C}^{1}(\mathbb{R} ; \mathbb{C}): x \text { is } 2 \pi \text { - periodic, }, \begin{array}{r}
x(t) \neq x(\theta), 0<|t-\theta|<2 \pi \\
\left.\left|x^{\prime}(\theta)\right| \neq 0, \forall \theta, \quad \text { ind } x^{\prime}=1\right\} .
\end{array}\right.
$$

This set corresponds to proper parametrizations of $\mathrm{C}^{1}$ simple closed curves in the complex plane. The fact that the winding number ind $\left(x^{\prime}\right)=1$ means that this curve is described once counterclockwise when the parameter grows over a period.

We also consider the function $G=\left(G_{1}, G_{2}, G_{3}\right)$ defined by

$$
\begin{aligned}
G_{1}(x, c, \lambda) & :=\frac{1}{2 \pi} \int_{0}^{2 \pi} \log |x(\cdot)-x(\theta)| \mathrm{d} \theta-\frac{1}{2 \pi} \int_{K} j(y) \log |x(\cdot)-\lambda y| \mathrm{d} y+c \\
G_{2}(x, c, \lambda) & :=\left(\left|x^{\prime}(\cdot)\right|^{2}-1\right) / 2 \\
G_{3}(x, c, \lambda) & :=x_{2}(0) .
\end{aligned}
$$

We turn back now to our problem and for simplicity we will further assume that $\Gamma$ is connected, i.e. $\Omega$ is a simply connected domain. We then represent the scaled curve $\lambda \Gamma$ (of total length $2 \pi$ ) by its $2 \pi$-periodized arclength parametrization $x: \mathbb{R} \rightarrow \mathbb{R}^{2} \equiv \mathbb{C}$ (satisfying then $x \in E$ and $\left|x^{\prime}(\theta)\right|=1$, i.e. $G_{2}(x, c, \lambda)=0$ ). From (3) it can be shown that $\int_{\Gamma} y_{k} \mathrm{~d} \sigma_{y}=0$ for $k=1,2$ and therefore $\Gamma$ crosses the $O y_{1}$ axis. Thus we can require that $x_{2}(0)=0$ so that $G_{3}(x, c, \lambda)=0$.

To even out the number of equations and unknowns we add a real parameter $c$ and consider the following problem:

$$
\left\{\begin{array}{l}
\text { find admissible } x \in E \text { and } c \in \mathbb{R} \text { such that } \\
G(x, c, \lambda)=0
\end{array}\right.
$$

By admissible we understand that $\lambda K$ is included in the bounded domain $\lambda \Omega$ delimited by the Jordan curve $\lambda \Gamma$ parametrized by $x$. If $\Omega$ is simply connected and $\partial \Omega=\Gamma \in \mathrm{C}^{1}$, then (4) is equivalent to (6), with $\lambda^{-1} x$ the arc parametrization of $\Gamma$. Let us prove shortly this equivalence. The only non trivial thing is to show that $c=0$ if $G_{1}(x, c, \lambda)=0$. For that we define a function $v$ by

$$
v(z)=\frac{1}{2 \pi} \int_{0}^{2 \pi} \log |z-x(\theta)| \mathrm{d} \theta-\frac{1}{2 \pi} \int_{K} \log |z-\lambda y| j(y) \mathrm{d} y+c,
$$


and remark that then $v=0$ on $\lambda \Gamma, \Delta v=0$ outside $\lambda \bar{\Omega}$ and $v(z)=c+O\left(|z|^{-1}\right)$ as $z \rightarrow \infty$. That implies $v=0$ outside $\lambda \Omega$ and consequently $c=0$.

We will work now with this condensed form (6) and will use that its solutions have the properties: $x$ is analytic and $c=0$.

Remark 2.1. It seems easy to adapt our analysis to the situation of two or more connected components of the boundary. The writting is a little more intricate but the theory could be applied as far as the components are separated. But we have failed in our attempt to analyse for instance the transition from a situation with one to another with two components as it occurs with the example of two Dirac masses described in Section 12.

\section{FunCtional SeTting}

To each complex or real valued $2 \pi$-periodic function $v$ defined on $\mathbb{R}$ we associate its Fourier representation

$$
v(t)=\sum_{k \in \mathbb{Z}} v_{k} e^{\imath k t} .
$$

We will use the Sobolev spaces (for $s \geq 0$ )

$$
\mathrm{H}^{s}:=\left\{v: \sum_{k \in \mathbb{Z}}|k|^{2 s}\left|v_{k}\right|^{2}<\infty\right\}
$$

endowed with the semi-norms and norms

$$
|v|_{s}^{2}=\sum_{k \in \mathbb{Z}}|k|^{2 s}\left|v_{k}\right|^{2} \quad \text { and, if } s>0, \quad\|v\|_{s}^{2}=\left|v_{0}\right|^{2}+|v|_{s}^{2} .
$$

For $s=0$ we set $\|v\|_{0}=|v|_{0}$. The set $\mathrm{H}^{0}$ coincides with $\mathrm{L}^{2}(0,2 \pi)$ (extended to $\mathbb{R}$ by periodicity). We will use the closed subset of the real valued functions of $\mathrm{H}^{s}$

$$
\mathrm{H}_{\mathbb{R}}^{s}:=\left\{v \in \mathrm{H}^{s}: v(\cdot) \in \mathbb{R}\right\}=\left\{v \in \mathrm{H}^{s}: v_{-k}=\bar{v}_{k}, \forall k\right\},
$$

which is a real vector space.

We will need to use the orthogonal projectors $P$ and $Q$ defined on $\mathrm{H}^{s}$ by

$$
P v(t):=\sum_{k \geq 0} v_{k} e^{\imath k t} \text { and } Q v(t):=\sum_{k<0} v_{k} e^{\imath k t} .
$$

We have clearly $P+Q=I d$. Recall that these projectors are related with the Hilbert transform $S$

$$
S v(t):=-\frac{1}{\pi} \int_{0}^{2 \pi} \frac{e^{\imath \theta} v(\theta)}{e^{\imath t}-e^{\imath \theta}} \mathrm{d} \theta
$$

by the relations $(c f .[15])$

$$
P=\frac{1}{2}(I d+S), \quad \text { and } \quad Q=\frac{1}{2}(I d-S) .
$$

We introduce finally the set

$$
\mathcal{U}_{s}:=\left\{(x, c, \lambda) \in \mathrm{H}^{s} \times \mathbb{R} \times \mathbb{R}: x \in E, \lambda K \subset D_{x}\right\},
$$

where $D_{x}$ denotes the bounded domain delimited by the curve described by $x$. It can be seen that, for $s>3 / 2$, $\mathcal{U}_{s}$ is an open subset of $\mathrm{H}^{s} \times \mathbb{R} \times \mathbb{R}$.

Theorem 3.1. For $s>3 / 2, G \in \mathrm{C}^{\infty}\left(\mathcal{U}_{s} ; \mathrm{H}_{\mathbb{R}}^{s} \times \mathrm{H}_{\mathbb{R}}^{s-1} \times \mathbb{R}\right)$. 
Proof. The condition $s>3 / 2$ implies that $\mathrm{H}^{s} \subset \mathrm{C}^{1}$ and that $\mathrm{H}^{s-1}$ is an algebra. Then it is easy to verify that the components $G_{2}$ and $G_{3}$ are $\mathrm{C}^{\infty}$. For the first component the only difficulty is to prove the $\mathrm{C}^{\infty}$ regularity of the map $\varphi$

$$
\begin{gathered}
\varphi(x)(t):=\int_{0}^{2 \pi} \log |x(t)-x(\theta)| \mathrm{d} \theta=\int_{0}^{2 \pi} \log |b(x)(t, \theta)| \mathrm{d} \theta \\
\text { where } \quad b(x)(t, \theta):=\frac{x(t)-x(\theta)}{e^{\imath t}-e^{\imath \theta}} .
\end{gathered}
$$

We need to introduce some Sobolev spaces of biperiodic functions

$$
\mathrm{H}_{b}^{\sigma}=\left\{v: v(t, \theta)=\sum_{k, l \in \mathbb{Z}} v_{k l} e^{\imath k t} e^{\imath l \theta}, \sum_{k, l \in \mathbb{Z}}\left(|k|^{2 \sigma}+|l|^{2 \sigma}\right)\left|v_{k l}\right|^{2}<\infty\right\} .
$$

It is easily seen that the linear operator $b$ is continuous from $\mathrm{H}^{s}$ into $\mathrm{H}_{b}^{s-1 / 2} \subset \mathrm{C}^{0}\left([0,2 \pi]^{2}\right)$. Furthermore $b(x)$ does not vanish if $x \in E$. Thus we conclude that the map $x \mapsto \log |b(x)|$ is $\mathrm{C}^{\infty}$ from $E \cap \mathrm{H}^{s}$ into $\mathrm{C}^{0}\left([0,2 \pi]^{2}\right)$ and consequently $\varphi$ is $\mathrm{C}^{\infty}$ from $E \cap \mathrm{H}^{s}$ into $\mathrm{C}^{0}([0,2 \pi])$.

A simple calculation shows that

$$
\frac{\mathrm{d}}{\mathrm{d} t} \varphi(x)=\operatorname{Re}\left(\alpha(x) x^{\prime}\right), \text { where } \alpha(x):=\int_{0}^{2 \pi} \frac{\mathrm{d} \theta}{x(\cdot)-x(\theta)},
$$

(the integral being understood in the Cauchy principal value sense). We introduce a linear operator $h$ by

$$
h(v)(t):=\int_{0}^{2 \pi} \frac{v(t, \theta)}{e^{\imath t}-e^{2 \theta}} \mathrm{d} \theta,
$$

so that clearly $\alpha(x)=h(1 / b(x))$. Since $b(x)$ does not vanish on $E \cap \mathrm{H}^{s}$, the map $x \mapsto 1 / b(x)$ is $\mathrm{C}^{\infty}$ from $E \cap \mathrm{H}^{s}$ into $\mathrm{H}_{b}^{s-1 / 2}$. The linear operator $h$ being continuous from $\mathrm{H}_{b}^{s-1 / 2}$ into $\mathrm{H}^{s-1}$, we deduce that $\alpha$ is $\mathrm{C}^{\infty}$ from $E \cap \mathrm{H}^{s}$ into $\mathrm{H}^{s-1}$. Coming back to (10) and using that $\mathrm{H}^{s-1}$ is an algebra we have proven that $x \mapsto(\varphi(x))^{\prime}$ is $\mathrm{C}^{\infty}$ from $E \cap \mathrm{H}^{s}$ into $\mathrm{H}^{s-1}$ and finally that $\varphi$ is $\mathrm{C}^{\infty}$ from $E \cap \mathrm{H}^{s}$ into $\mathrm{H}^{s}$.

In order to avoid the difficulty of mixing $\mathbb{C}-$ and $\mathbb{R}$-spaces, we will consider the variable $\underline{x}:=(x, c)=$ $\left(x_{1}, x_{2}, c\right)$ as belonging to $\mathrm{H}_{\mathbb{R}}^{s} \times \mathrm{H}_{\mathbb{R}}^{s} \times \mathbb{R}$ and, for the sake of simplicity, we will denote by $\mathrm{D}$ the (real) Fréchet derivative operator with respect to this variable $\underline{x}$. By Theorem $3.1, \mathrm{D} G(\underline{x}, \lambda)$ is well defined for all $(\underline{x}, \lambda) \in \mathcal{U}_{s}$ if $s>3 / 2$.

\section{Some properties and particular CASES}

\subsection{A particular case: $\lambda=0$}

Let us assume that $s>3 / 2$. By the previous theory we know that given $\lambda>0$, every solution of

$$
\left\{\begin{array}{l}
(x, c, \lambda) \in \mathcal{U}_{s} \\
G(x, c, \lambda)=0
\end{array}\right.
$$

satisfies that $c=0$ and $x$ is analytic. If we look back to problem (1), the normalization (2) excludes the possibility of having $\lambda<0$, and the case $\lambda=0$ is not compatible with a bounded domain $\Omega$. These restrictions do not occur with problem (11) (this is due to the scaling). In particular we will see that the case $\lambda=0$ is of special interest. For that we introduce a function

$$
x_{0}(t)=e^{\imath t}, \quad \forall t, \quad \underline{x}_{0}=\left(x_{0}, 0\right) .
$$


It is an easy matter to verify that $G\left(x_{0}, 0,0\right)=0$ (we also have $G\left(-x_{0}, 0,0\right)=0$ ). Using formula (9) we obtain by a simple calculation that the derivative $A_{0}:=\mathrm{D} G\left(x_{0}, 0,0\right)$ is given by (recall that $Q$ is defined by (8))

$$
A_{0}(y, \gamma)=\left(-\operatorname{Re} Q\left(\bar{x}_{0} y\right)+\gamma, \operatorname{Im}\left(\bar{x}_{0} y^{\prime}\right), \operatorname{Im} y(0)\right)
$$

Proposition 4.1. If $\lambda=0$, the problem (11) has two solutions: $\left(x_{0}, 0\right)$ and $-\left(x_{0}, 0\right)$. Moreover the operator $A_{0}:=\mathrm{D} G\left(x_{0}, 0,0\right)$ is an isomorphism from $X^{\sigma}$ onto $Y^{\sigma}$ for all $\sigma>1 / 2$, where

$$
X^{\sigma}:=\mathrm{H}_{\mathbb{R}}^{\sigma} \times \mathrm{H}_{\mathbb{R}}^{\sigma} \times \mathbb{R} \quad \text { and } \quad Y^{\sigma}:=\mathrm{H}_{\mathbb{R}}^{\sigma} \times \mathrm{H}_{\mathbb{R}}^{\sigma-1} \times \mathbb{R}
$$

Proof. We write $y=x_{0} z \in \mathrm{H}^{\sigma}, f_{1} \in \mathrm{H}_{\mathbb{R}}^{\sigma}, f_{2} \in \mathrm{H}_{\mathbb{R}}^{\sigma-1}$ on Fourier form

$$
z(t)=\sum_{k \in \mathbb{Z}} z_{k} e^{\imath k t}, \quad f_{1}(t)=\sum_{k \in \mathbb{Z}} \alpha_{k} e^{\imath k t}, \quad f_{2}(t)=\sum_{k \in \mathbb{Z}} \beta_{k} e^{\imath k t},
$$

with $\alpha_{-k}=\bar{\alpha}_{k}$ and $\beta_{-k}=\bar{\beta}_{k}$. Then the relation $A_{0}(y, \gamma)=\left(f_{1}, f_{2}, \delta\right)$ is equivalent to

$$
\left\{\begin{array}{lll}
\text { for } k \geq 1, & z_{-k}=-2 \bar{\alpha}_{k}, & (k+1) z_{k}=\beta_{k}+2(k-1) \alpha_{k}, \\
\gamma=\alpha_{0}, & \operatorname{Re} z_{0}=\beta_{0} / 2, & \operatorname{Im} z_{0}=d-\operatorname{Im} \sum_{k \neq 0} z_{k} .
\end{array}\right.
$$

That proves the existence of $A_{0}^{-1}$ and its continuity, the condition $\sigma>1 / 2$ being used to ensure the convergence of the series

$$
\sum_{k \neq 0}\left|z_{k}\right| \leq \sum_{k>0}\left(4\left|\alpha_{k}\right|+\left|\beta_{k}\right| / k\right) \leq\left(\sum_{k>0} k^{-2 \sigma}\right)^{1 / 2}\left(\sum_{k>0}\left(4 k^{\sigma}\left|\alpha_{k}\right|+k^{\sigma-1}\left|\beta_{k}\right|\right)^{2}\right)^{1 / 2} .
$$

Remark 4.2. These two solutions represent in fact the same curve (the unit circle) in the complex plane. It can be shown [5] that they are the only solutions. From this proposition we deduce that problem (11) has a regular branch of solutions in a neighborhood of $\lambda=0$. If we come back to the initial problem we deduce that, for $\lambda>0$ small enough, the problem (1) has a solution $\partial \Omega$ whose the behaviour is asymptotically that of the circle centered at the origin of radius $1 / \lambda$.

\subsection{The Fredholm character of the derivative}

The simple form of $\mathrm{D} G$ for $\lambda=0$ allows a convenient representation of this derivative on the solution in all other cases. If we explicit the first component of $\mathrm{D} G$ we get

$$
\mathrm{DG}_{1}(x, c, \lambda)(y, \gamma)=\frac{1}{2 \pi} \operatorname{Re}\left[\int_{0}^{2 \pi} \frac{y(\cdot)-y(\theta)}{x(\cdot)-x(\theta)} \mathrm{d} \theta-y(\cdot) \int_{K} \frac{j(\xi)}{x(\cdot)-\lambda \xi} \mathrm{d} \xi\right]+\gamma
$$

If $\underline{x}$ is a solution of (11) then we have seen that $c=0, x$ is analytic and the function $v$ defined by (7) satisfies $v=0$ outside $\lambda \bar{\Omega}$. Thus we deduce that

$$
\pi\left(\partial_{z_{1}} v-\imath \partial_{z_{2}} v\right)=\int_{0}^{2 \pi} \frac{1}{z-x(\theta)} \mathrm{d} \theta-\int_{K} \frac{j(\xi)}{z-\lambda \xi} \mathrm{d} \xi=0, \quad \text { outside } \lambda \bar{\Omega} .
$$

Taking the limit as $z$ tends to $x(\cdot) \in \lambda \Gamma$ and using the result in (13) we deduce the following expression for $\mathrm{DG}$ (on a solution)

$$
\mathrm{D} G_{1}(x, c, \lambda)(y, \gamma)=-\frac{1}{2 \pi} \operatorname{Re}\left[\int_{0}^{2 \pi} \frac{y(\theta)}{x(\cdot)-x(\theta)} \mathrm{d} \theta+\pi \imath \frac{y(\cdot)}{x^{\prime}(\cdot)}\right]+\gamma
$$




$$
\mathrm{D} G_{2}(x, c, \lambda)(y, \gamma)=\operatorname{Re} \bar{x}^{\prime}(\cdot) y^{\prime}(\cdot), \quad \text { and } \quad \mathrm{DG}_{3}(x, c, \lambda)(y, \gamma)=\operatorname{Im} y(0)
$$

We now introduce a linear operator $L$ and a function $a$ by

$$
L(y, \gamma):=(a y, \gamma) \text { and } a:=x_{0}^{\prime} / x^{\prime}
$$

and we set

$$
K:=\mathrm{D} G(x, 0, \lambda)-A_{0} L
$$

We are able to state the following result.

Proposition 4.3. If $(x, 0, \lambda)$ is a solution to (11) and $\sigma>1 / 2$, then

$$
\mathrm{D} G(x, 0, \lambda)=A_{0} L+K
$$

where $L$ is an automorphism of $X^{\sigma}$, and $K$ is a compact operator from $X^{\sigma}$ into $Y^{\sigma}$.

Proof. We remark first that $a$ is analytic and satisfies $|a|=1$ since $\underline{x}$ is a solution of (11). Therefore $L$ is clearly an automorphism. We now successively look at the different components of $K$. We deduce from (15)

$$
K_{1}(y, \gamma)=\frac{1}{2 \pi} \operatorname{Re} \int_{0}^{2 \pi}\left[-\frac{y(\theta)}{x(\cdot)-x(\theta)}+\frac{a(\theta) y(\theta)}{x_{0}(\cdot)-x_{0}(\theta)}\right] \mathrm{d} \theta
$$

and thus

$$
K_{1}(y, \gamma)=\frac{1}{2 \pi} \operatorname{Re} \int_{0}^{2 \pi} k(\cdot, \theta) y(\theta) \mathrm{d} \theta, \quad \text { with } k(t, \theta):=\frac{x_{0}^{\prime}(\theta) / x^{\prime}(\theta)}{x_{0}(t)-x_{0}(\theta)}-\frac{1}{x(t)-x(\theta)}
$$

Since $x$ is analytic and $x^{\prime}$ does not vanish we deduce that $k \in \mathrm{C}^{\infty}\left([0,2 \pi]^{2}\right)$ and is $2 \pi$-periodic in both variables. This implies that $K_{1}: X^{\sigma} \rightarrow \mathrm{H}^{s}$ is a linear compact operator for all $s$.

Simple calculations give $K_{2}(y, \gamma)=-\operatorname{Re}\left(\bar{x}_{0}^{\prime} a^{\prime} y\right)$ and $K_{3}(y, \gamma)=\operatorname{Im}[(1-a(0)) y(0)]$. Therefore $K_{2}$ is linear continuous from $X^{\sigma}$ into $H_{\mathbb{R}}^{\sigma}$ and compact into $H_{\mathbb{R}}^{\sigma-1}$. The case of $K_{3}$ is straightforward.

Remark 4.4. This proposition implies that $\mathrm{D} G(x, 0, \lambda)$ is a Fredholm operator of index 0 from $X^{\sigma}$ into $Y^{\sigma}$. The compactness of $K$ will play a crucial role for the numerical analysis of the problem. In view of the trigonometrical approximation it will be important also to remark that the function $a$ satisfies

$$
|a|=1 \quad \text { and } \quad \text { ind } a=0 \text {. }
$$

\subsection{The convex case}

When the solution $\Omega$ of problem (1) is convex some uniqueness results are known $[7,13]$. In our context we have the following statement which implies local uniqueness.

Proposition 4.5. We assume that $(x, 0, \lambda)$ is a solution of $(11)$ and that the curve described by $x$ is convex. Then $\mathrm{D} G(x, 0, \lambda)$ is an isomorphism of $X^{\sigma}$ into $Y^{\sigma}$ for all $\sigma>1 / 2$.

Proof. We will denote here DG $:=\mathrm{D} G(x, 0, \lambda)$. Let us consider $(y, \gamma) \in \operatorname{Ker} \mathrm{D} G$. From the previous proposition it is sufficient to prove that $(y, \gamma)=0$. We first deduce from $A_{0} L(y, \gamma)=-K(y, \gamma)$ and from the regularising properties of $K$ that $y \in \mathrm{C}^{\infty}$. Then we define the real valued functions $\alpha, \beta$ and $\kappa$ by

$$
\alpha+\imath \beta:=\bar{x}^{\prime} y \quad \text { and } \quad \kappa:=\imath x^{\prime} \bar{x}^{\prime \prime} \text {. }
$$


Recall that we have $x^{\prime} \bar{x}^{\prime}=1$ and $\kappa(t)$ represents the curvature at the point $x(t)$. From the convexity assumption the values of $\kappa$ are thus positive or null. We deduce from $0=\mathrm{D} G_{2}(y, \gamma)=\operatorname{Re} \bar{x}^{\prime} y^{\prime}$ that

$$
\alpha^{\prime}=\kappa \beta .
$$

Let $\omega$ be the bounded domain delimited by $x$ and

$$
v(z):=-\frac{1}{2 \pi} \int_{0}^{2 \pi} \frac{y(\theta)}{z-x(\theta)} \mathrm{d} \theta+\gamma, \quad z \in \omega
$$

which admits the continuous extension on $\partial \omega$

$$
v(x(\cdot)):=-\frac{1}{2 \pi} \int_{0}^{2 \pi} \frac{y(\theta)}{x(\cdot)-x(\theta)} \mathrm{d} \theta+\gamma+\frac{i}{2} \frac{y(\cdot)}{x^{\prime}(\cdot)} .
$$

From the assumption $\mathrm{D} G_{1}(y, \gamma)=0$ we deduce that $v(x)=\imath \bar{x}^{\prime} y=\imath(\alpha+\imath \beta)$ on $\partial \omega$. Using now Green's formula for the holomorphic function $v$ we obtain

$$
\left.\int_{\omega}\left|v^{\prime}(z)\right|^{2} \mathrm{~d} z=\frac{\imath}{2} \int_{0}^{2 \pi} v(x(t)) \overline{v(x(t)}\right)^{\prime} \mathrm{d} t=-\int_{0}^{2 \pi} \alpha^{\prime}(t) \beta(t) \mathrm{d} t .
$$

Taking into account (16) and $\kappa \geq 0$, we obtain $\kappa \beta=0$ on $\mathbb{R}$ and $v^{\prime}=0$ in $\omega$. Going to the boundary we deduce that $v(x)=\imath \alpha-\beta$ is constant on $\mathbb{R}$. Since $\kappa$ does not vanish identically, the real $\beta$ is $0, \alpha$ is constant and $y=\alpha x^{\prime}$. We get $\alpha=0$ and thus $y=0$ from the last component $0=\mathrm{D} G_{3}(y, \gamma)=\alpha x_{2}^{\prime}(0)$ since $x_{2}^{\prime}(0) \neq 0$. Coming back to $\mathrm{D} G_{1}(y, \gamma)=0$ we deduce finally $\gamma=0$.

It remains to prove that $x_{2}^{\prime}(0) \neq 0$. Indeed otherwise the boundary $\partial \omega$ would be tangent to the real axis at the point $x(0)$ and therefore the convex $\omega$ would be located on only one side of this axis. This fact is uncompatible with the relation $\int x_{2} \mathrm{~d} t=0$ which follows from the assumption (3).

\section{General approximation theory}

Here as in Sections 8 to 11 we take a fixed value of $\lambda$ and avoid reference to this parameter in all functions in order to lighten notations. Section 6 deals with dependence on $\lambda$.

Let then $\mathcal{U}:=\left\{\underline{x}=(x, c):(x, c, \lambda) \in \mathcal{U}_{2}\right\}$. We equally denote $G \underline{x}:=G(x, c, \lambda)$. In this section we are concerned with the numerical approximation of

$$
\underline{x} \in \mathcal{U}, \quad G \underline{x}=0,
$$

by a general projection method. Let $h$ be a positive real parameter, $X_{h}=X_{h, 0} \times \mathbb{R} \subset X:=\mathrm{H}^{2} \times \mathbb{R}$ and $Y_{h} \subset Y:=\mathrm{H}_{\mathbb{R}}^{2} \times \mathrm{H}_{\mathbb{R}}^{1} \times \mathbb{R}$ be finite dimensional spaces of the same dimension and let $P_{h}: Y \rightarrow Y_{h}$ be a linear projection onto $Y_{h}$. Thus we consider the general discrete problem

$$
\underline{x}_{h} \in X_{h}, \quad P_{h} G \underline{x}_{h}=0,
$$

which is equivalent to a system of nonlinear equations.

In the following sections we will give more precise details on how to adequately write our discrete methods in form (18). We will be dealing with the following four families of numerical methods. 
(a) Trigonometric Galerkin methods. Let $n$ be a positive integer, $h:=1 / n$. We introduce the space $\mathbb{T}_{n}$ of trigonometric polynomials with degree $\leq n$ and the orthogonal projector $\pi_{n}$ on $\mathbb{T}_{n}$

$$
\mathbb{T}_{n}:=\left\{p: p(t)=\sum_{k=-n}^{n} p_{k} e^{\imath k t}, \quad p_{k} \in \mathbb{C}\right\}, \quad \pi_{n} v(t):=\sum_{k=-n}^{n} v_{k} e^{\imath k t}
$$

Notice that if $v \in \mathrm{H}_{\mathbb{R}}^{0}$, then $\pi_{n} v$ is a real valued trigonometric polynomial. We define $X_{h}:=x_{0} \mathbb{T}_{n} \times \mathbb{R}$ (recall that $x_{0}(t)=e^{\imath t}$ ) and consider the numerical method

$$
\left\{\begin{array}{l}
\text { Find } \underline{x}_{h} \in X_{h}, \quad \text { such that } \\
\left(\pi_{n} G_{1} \underline{x}_{h}, \pi_{n} G_{2} \underline{x}_{h}, G_{3} \underline{x}_{h}\right)=(0,0,0)
\end{array}\right.
$$

(b) Trigonometric collocation. Let $X_{h}$ be as in (a). For $j=1,2$ take arbitrary $\theta_{0}^{j}$ and define $\theta_{k}^{j}:=$ $\theta_{0}^{j}+2 k \pi /(2 n+1)$ for $k \neq 0$. We then consider the discrete problem, which is equivalent to a nonlinear system of $4 n+3$ equations

$$
\left\{\begin{array}{l}
\text { Find } \underline{x}_{h} \in X_{h}, \quad \text { such that } \\
G_{1} \underline{x}_{h}\left(\theta_{k}^{1}\right)=0, \quad \forall k, \quad G_{2} \underline{x}_{h}\left(\theta_{k}^{2}\right)=0, \quad \forall k, \quad G_{3} \underline{x}_{h}=0 .
\end{array}\right.
$$

(c) Spline Galerkin method. Let $\mathbb{P}_{d}$ be the space of complex-valued polynomials of degree at most $d$. Let $\Delta_{h}$ denote a partition

$$
t_{0}<t_{1}<\ldots<t_{n}=t_{0}+2 \pi
$$

extended by $2 \pi$-periodicity to $\left\{t_{j}: j \in \mathbb{Z}\right\}, h=\max _{j}\left|t_{j+1}-t_{j}\right|$. For $d \geq 1$, let

$$
S^{d}\left(\Delta_{h}\right):=\left\{u \in \mathrm{H}^{0}: u \in \mathrm{C}^{d-1},\left.u\right|_{\left(t_{j}, t_{j+1}\right)} \in \mathbb{P}_{d}, \forall j\right\}
$$

be the space of smoothest splines of degree $d$ and let

$$
S_{\mathbb{R}}^{d}\left(\Delta_{h}\right):=S^{d}\left(\Delta_{h}\right) \cap \mathrm{H}_{\mathbb{R}}^{0}=\left\{x_{h} \in S^{d}\left(\Delta_{h}\right): x_{h}(\cdot) \in \mathbb{R}\right\} .
$$

In order to have $S^{d}(\Delta) \subset \mathrm{H}^{2}$ we assume henceforth $d \geq 2$ and consider the method

$$
\begin{cases}\text { Find } \underline{x}_{h} \in S^{d}\left(\Delta_{h}\right) \times \mathbb{R}, & \text { such that } \\ \left(G_{1} \underline{x}_{h}, y_{h}\right)=0, & \forall y_{h} \in S_{\mathbb{R}}^{d}\left(\Delta_{h}\right), \\ \left(G_{2} \underline{x}_{h}, z_{h}\right)=0, & \forall z_{h} \in S_{\mathbb{R}}^{d-1}\left(\Delta_{h}\right), \\ G_{3} \underline{x}_{h}=0, & \end{cases}
$$

where $(f, g):=\int_{0}^{2 \pi} f(t) g(t) \mathrm{d} t$ denotes the inner product in $\mathrm{H}_{\mathbb{R}}^{0}$.

(d) Spline collocation. Consider $X_{h}:=S^{d}\left(\Delta_{h}\right) \times \mathbb{R}$ as in (c). If $d$ is even let

$$
\theta_{k}^{1}:=\left(t_{k}+t_{k+1}\right) / 2, \quad \theta_{k}^{2}:=t_{k},
$$

whereas if $d$ is odd, we reverse the roles of $\theta_{k}^{1}$ and $\theta_{k}^{2}$. We then consider the scheme (19) with these new definitions.

To begin with the analysis of the method (18) we assume the following hypothesis.

Hypothesis (H1). The family of operators $P_{h}: Y \rightarrow Y_{h} \subset Y$ is uniformly bounded. 
Let $G_{h}:=P_{h} G$. If $\underline{x}$ is the exact solution to (17), we define

$$
A_{h}:=\left.P_{h} \mathrm{D} G(\underline{x})\right|_{X_{h}}=\left.\mathrm{D} G_{h}(\underline{x})\right|_{X_{h}}: X_{h} \subset X \rightarrow Y_{h} \subset Y .
$$

We also assume the following hypothesis. Its verification for the examples above will take most of the work in Sections to come.

Hypothesis (H2). There exists $h_{0}>0$ and $C$ independent of $h$ such that for all $h \leq h_{0}$

$$
\left\|\underline{z}_{h}\right\|_{X} \leq C\left\|A_{h} \underline{z}_{h}\right\|_{Y}, \quad \forall \underline{z}_{h} \in X_{h} .
$$

Obviously (H2) implies the existence of $A_{h}^{-1}$ and its uniform boundedness.

Lemma 5.1. If (H1) and (H2) hold, there exists $\alpha>0$ such that, for all $h \leq h_{0}$ and all $\underline{z} \in X$ such that $\|\underline{z}-\underline{x}\|_{X} \leq \alpha$,

$$
\left\|A_{h}^{-1} \mathrm{D}^{2} G_{h}(\underline{z})\right\|_{\mathcal{L}(X \times X ; X)} \leq 1 / 2 \alpha .
$$

Proof. Indeed from (H1-H2) we have

$$
\left\|A_{h}^{-1} \mathrm{D}^{2} G_{h}(\underline{z})\right\|_{\mathcal{L}(X \times X ; X)} \leq C\left\|\mathrm{D}^{2} G(\underline{z})\right\|_{\mathcal{L}(X \times X ; Y)} .
$$

Thus the lemma follows from the continuity of the map $\underline{z} \mapsto \mathrm{D}^{2} G_{h}(\underline{z})$.

To prove existence and uniqueness of solution to (18), as well as some convergence estimates, we transform problem (18) into a fixed point problem

$$
\underline{x}_{h}=\Phi_{h} \underline{x}_{h},
$$

where

$$
\Phi_{h}:=\left.\left(I-A_{h}^{-1} G_{h}\right)\right|_{X_{h}},
$$

i.e., we consider a modification of Newton's method. For the value of $\alpha$ determined by Lemma 5.1, let

$$
B_{h}:=\left\{\underline{z} \in X_{h}:\|\underline{z}-\underline{x}\|_{X} \leq \alpha\right\} .
$$

Lemma 5.2. For all $h \leq h_{0}$ and $\underline{z}_{1}, \underline{z}_{2} \in B_{h}$,

$$
\left\|\Phi_{h \underline{z}_{1}}-\Phi_{h \underline{z}_{2}}\right\|_{X} \leq \frac{1}{2}\left\|\underline{z}_{1}-\underline{z}_{2}\right\|_{X} .
$$

Proof. By Taylor's formula we have

$$
\begin{aligned}
\Phi_{h \underline{z}_{1}-\Phi_{h} \underline{z}_{2}} & =A_{h}^{-1}\left(\mathrm{D} G_{h}(\underline{x})\left(\underline{z}_{1}-\underline{z}_{2}\right)-G_{h} \underline{z}_{1}+G_{h} \underline{z}_{2}\right) \\
& =A_{h}^{-1}\left[\int_{0}^{1}\left(\mathrm{D} G_{h}(\underline{x})-\mathrm{D} G_{h}\left(t \underline{z}_{1}+(1-t) \underline{z}_{2}\right)\right) \mathrm{d} t\right]\left(\underline{z}_{1}-\underline{z}_{2}\right) .
\end{aligned}
$$

On the other hand if $\|\underline{\zeta}-\underline{x}\|_{X}<\alpha$, by Proposition 5.1

$$
\begin{aligned}
\left\|A_{h}^{-1}\left(\mathrm{D} G_{h}(\underline{x})-\mathrm{D} G_{h}(\underline{\zeta})\right)\right\|_{\mathcal{L}(X ; X)} & =\left\|A_{h}^{-1} \int_{0}^{1} \mathrm{D}^{2} G_{h}((1-\tau) \underline{x}+\tau \underline{\zeta}) \mathrm{d} \tau(\underline{x}-\underline{\zeta})\right\|_{\mathcal{L}(X ; X)} \\
& \leq \frac{1}{2 \alpha}\|\underline{x}-\underline{\zeta}\|_{X} \leq \frac{1}{2} .
\end{aligned}
$$

Since for all $t \in[0,1], \underline{z}_{1}+(1-t) \underline{z}_{2} \in B_{h},(20)$ and (21) prove the result. 
Lemma 5.3. If (H1) and (H2) hold, then there exists $K>0$ such that, for all $h \leq h_{0}$ and $\underline{z} \in B_{h}$,

$$
\left\|A_{h}^{-1} G_{h} \underline{z}\right\|_{X} \leq K\|\underline{x}-\underline{z}\|_{X} .
$$

Proof. Take $\underline{z} \in B_{h}$. By a new application of the Taylor expansion and by Lemma 5.1, we obtain

$$
\begin{aligned}
\left\|A_{h}^{-1} G_{h} \underline{z}\right\|_{X} & =\left\|A_{h}^{-1}\left(G_{h} \underline{x}-G_{h} \underline{z}\right)\right\|_{X} \\
& \leq\left\|A_{h}^{-1} \mathrm{D} G_{h}(\underline{x})\right\|_{\mathcal{L}(X ; X)}\|\underline{x}-\underline{z}\|_{X}+\frac{1}{4 \alpha}\|\underline{x}-\underline{z}\|_{X}^{2} .
\end{aligned}
$$

Then (H2) implies that

$$
\left\|A_{h}^{-1} G_{h} \underline{z}\right\|_{X} \leq\left(C\left\|\mathrm{D} G_{h}(\underline{x})\right\|_{\mathcal{L}(X ; Y)}+\frac{1}{4}\right)\|\underline{x}-\underline{z}\|_{X} .
$$

Hence (H1) gives the result.

Hypothesis (H3). There exists a family of operators $\widetilde{P}_{h}: X \rightarrow X_{h}$ such that

$$
\left\|\widetilde{P}_{h} \underline{x}-\underline{x}\right\|_{X} \rightarrow 0, \quad(\text { as } h \rightarrow 0) .
$$

Lemma 5.4. If (H1), (H2) and (H3) hold, then there exists $h_{1}>0$ such that for all $h \leq h_{1}$ the application $\Phi_{h}$ maps $B_{h}$ into itself.

Proof. By Lemma 5.3 and (H3) it follows that there exists $h_{1}$ such that for all $h \leq h_{1}, \widetilde{P}_{h} \underline{x} \in B_{h}$ and

$$
\frac{3}{2}\left\|\underline{x}-\widetilde{P}_{h} \underline{x}\right\|_{X}+\left\|A_{h}^{-1} G_{h} \widetilde{P}_{h} \underline{x}\right\|_{X} \leq \frac{\alpha}{2} .
$$

Let $\underline{z}_{h} \in B_{h}$. Then

$$
\left\|\Phi_{h} \underline{z}_{h}-\underline{x}\right\|_{X} \leq\left\|\Phi_{h} \underline{z}_{h}-\Phi_{h} \widetilde{P}_{h} \underline{x}\right\|_{X}+\left\|\widetilde{P}_{h} \underline{x}-\underline{x}\right\|_{X}+\left\|A_{h}^{-1} G_{h} \widetilde{P}_{h} \underline{x}\right\|_{X}
$$

Finally, by Lemma 5.2 and (22)

$$
\begin{aligned}
\left\|\Phi_{h \underline{z}_{h}}-\underline{x}\right\|_{X} & \leq \frac{1}{2}\left\|\underline{z}_{h}-\widetilde{P}_{h} \underline{x}\right\|_{X}+\left\|\widetilde{P}_{h} \underline{x}-\underline{x}\right\|_{X}+\left\|A_{h}^{-1} G_{h} \widetilde{P}_{h} \underline{x}\right\|_{X} \\
& \leq \frac{1}{2}\left\|\underline{z}_{h}-\underline{x}\right\|_{X}+\frac{\alpha}{2} \leq \alpha,
\end{aligned}
$$

from where the result follows.

Theorem 5.5. Assume that (H1) to (H3) hold. Then there exist $h_{1}>0$ and $\alpha>0$ such that (18) has a unique solution in $B_{h}$ for all $h \leq h_{1}$. Moreover, there exists a constant $K$ such that

$$
\left\|\underline{x}_{h}-\underline{x}\right\|_{X} \leq(2 K+1)\left\|\widetilde{P}_{h} \underline{x}-\underline{x}\right\|_{X},
$$

and therefore the method converges.

Proof. Existence and uniqueness of local solution follow from the application of Banach fixed point theorem to the problem $\underline{x}_{h}=\Phi_{h} \underline{x}_{h}$ by Lemmas 5.2 and 5.4. Also by Lemma 5.2

$$
\left\|\underline{x}_{h}-\widetilde{P}_{h} \underline{x}\right\|_{X} \leq \frac{1}{2}\left\|\underline{x}_{h}-\widetilde{P}_{h} \underline{x}\right\|_{X}+\left\|A_{h}^{-1} G_{h} \widetilde{P}_{h} \underline{x}\right\|_{X} .
$$

Therefore,

$$
\left\|\underline{x}_{h}-\underline{x}\right\|_{X} \leq\left\|\underline{x}-\widetilde{P}_{h} \underline{x}\right\|_{X}+2\left\|A_{h}^{-1} G_{h} \widetilde{P}_{h} \underline{x}\right\|_{X}
$$

and the result follows by Lemma 5.3. 
We finish this section by showing an equivalent form of Hypothesis (H2) to be used for our examples, under an additional more stringent condition on $P_{h}$. To simplify notations we write

$$
A:=\mathrm{D} G(\underline{x}): X \rightarrow Y .
$$

and recall the decomposition $A=A_{0} L+K$ given in (15), where $K$ is compact.

In the remainder of the paper, the arrow $\rightarrow$ will denote a weak limit and $\rightarrow$ a (strong) limit in the corresponding Hilbert space.

Lemma 5.6. Assume that $(\mathrm{H} 1)$ holds and:

(a) $y_{h}$ bounded and $P_{h} y_{h} \rightarrow 0$ in $Y \quad \Longrightarrow \quad y_{h} \rightarrow 0$ in $Y \quad($ as $h \rightarrow 0)$.

Then Hypothesis (H2) is equivalent to the following property:

(b) for all family $\left\{z_{h}\right\}_{h \in \mathcal{F}}$ such that $\left(z_{h}, 0\right) \in X_{h} \forall h$ we have, if $h \rightarrow 0$ in $\mathcal{F}$,

$$
z_{h} \rightarrow 0 \text { in } \mathrm{H}^{2} \text { and } P_{h} A_{0}\left(a z_{h}, 0\right) \rightarrow 0 \text { in } Y \quad \Longrightarrow \quad z_{h} \rightarrow 0 \text { in } \mathrm{H}^{2} \text {. }
$$

Proof. Assume that (H2) holds and let $\left(z_{h}, 0\right) \in X_{h}$ satisfy the requirements of (b). Since $K$ is compact and $P_{h}$ is uniformly bounded it follows that $\left\|A_{h}\left(z_{h}, 0\right)\right\|_{Y}=\left\|P_{h} A_{0}\left(a z_{h}, 0\right)+P_{h} K\left(z_{h}, 0\right)\right\|_{Y} \rightarrow 0$. Therefore (H2) implies $z_{h} \rightarrow 0$ in $\mathrm{H}^{2}$.

Let us now suppose that (H2) is false. Then we can take a family of $\left(z_{h}, \gamma_{h}\right) \in X_{h}$ such that

$$
\max \left(\left\|z_{h}\right\|_{2},\left|\gamma_{h}\right|\right)=1, \quad\left\|A_{h}\left(z_{h}, \gamma_{h}\right)\right\|_{Y} \rightarrow 0
$$

Then $y_{h}=A\left(z_{h}, \gamma_{h}\right)$ is bounded in $Y$ and $P_{h} y_{h} \rightarrow 0$ in $Y$. It follows from (a) that $y_{h} \rightarrow 0$ in $Y$. Since $A$ is an isomorphism we have $z_{h} \rightarrow 0$ in $\mathrm{H}^{2}$ and $\gamma_{h} \rightarrow 0$ in $\mathbb{R}$. By compactness of $K$ we get that $P_{h} A_{0}\left(a z_{h}, 0\right)=$ $A_{h}\left(z_{h}, \gamma_{h}\right)-A_{h}\left(0, \gamma_{h}\right)-P_{h} K\left(z_{h}, 0\right) \rightarrow 0$ in $Y$. But we have $\left\|z_{h}\right\|_{2}=1$ for $h$ small enough, and thus property (b) cannot hold.

Remark 5.7. Assume that there exist a function $\sigma$ and a Hilbert space $Z$ satisfying $Y \subset Z$ with continuous imbedding, such that

(a') $\quad \forall y \in Y, \quad\left\|y-P_{h} y\right\|_{z} \leq \sigma(h)\|y\|_{Y} \quad$ and $\quad \lim _{h \rightarrow 0} \sigma(h)=0$.

Then property (a) of the preceding lemma holds. Indeed let us consider a family $y_{h}$ bounded in $Y$ such that $P_{h} y_{h} \rightarrow 0$ in $Y$ and let $y$ be a weak limit of $y_{h}$ in $Y$. Then we have $y_{h} \rightarrow y$ in $Z$ but we have also $y_{h}-P_{h} y_{h} \rightarrow 0$ and $P_{h} y_{h} \rightarrow 0$ in $Z$, therefore $y=0$.

\section{UNIFORM APPROXIMATION ON A REGULAR BRANCH}

Consider the general problem

$$
\underline{x}_{\lambda} \in X, \quad G\left(\underline{x}_{\lambda}, \lambda\right)=0
$$

and its discretizations

$$
\underline{x}_{h, \lambda} \in X_{h}, \quad P_{h} G\left(\underline{x}_{h, \lambda}, \lambda\right)=0,
$$

following the ideas of Section 5. Assume that we are placed on a regular branch of the solution, i.e., that for all $\lambda \in[a, b]$ we can choose a solution to (23) such that $\lambda \mapsto \underline{x}_{\lambda}$ is a continuous function and that $D G\left(\underline{x}_{\lambda}, \lambda\right)$ is an isomorphism from $X$ onto $Y$. Therefore the map assigning $\underline{x}_{\lambda}$ to the parameter $\lambda$ is smooth.

Denote

$$
A_{h, \lambda}:=\left.\mathrm{D} G_{h}\left(\underline{x}_{\lambda}, \lambda\right)\right|_{X_{h}}
$$

with $G_{h}:=P_{h} G$ as before. The following hypotheses are generalizations of (H2) and (H3). 
Hypothesis (H2b). There exist $h_{0}>0$ and $C$ independent of $h$ and of $\lambda$ such that for all $h \leq h_{0}$ and $\lambda \in[a, b]$

$$
\left\|\underline{z}_{h}\right\|_{X} \leq C\left\|A_{h, \lambda} \underline{z}_{h}\right\|_{Y}, \quad \forall \underline{z}_{h} \in X_{h}
$$

Hypothesis (H3b). There exists a family of operators $\widetilde{P}_{h}: X \rightarrow X_{h}$ such that

$$
\max _{a \leq \lambda \leq b}\left\|\widetilde{P}_{h} \underline{x}_{\lambda}-\underline{x}_{\lambda}\right\|_{X} \rightarrow 0
$$

Theorem 6.1. If $(\mathrm{H} 1),(\mathrm{H} 2 \mathrm{~b})$ and $(\mathrm{H} 3 \mathrm{~b})$ hold, then we can take $h_{1}>0, \alpha$ and $K$ independent of $\lambda \in[a, b]$ in Theorem 5.5.

Proof. It follows readily that there exists $\alpha>0$ such that for all $\lambda \in[a, b]$ and for all $\underline{z} \in X$ such that $\left\|\underline{z}-\underline{x}_{\lambda}\right\|_{X}<\alpha$

$$
\left\|A_{h, \lambda}^{-1} \mathrm{D}^{2} G_{h, \lambda} \underline{z}\right\|_{\mathcal{L}(X \times X ; X)} \leq 1 / 2 \alpha, \quad \forall h \leq h_{0}
$$

where $G_{h, \lambda}:=P_{h} G(\cdot, \lambda)$. We then define $\Phi_{h, \lambda}:=\left.\left(I-A_{h, \lambda}^{-1} G_{h, \lambda}\right)\right|_{X_{h}}$. We can then follow all the steps in the proofs of Lemmas 5.3 and 5.4 and of Theorem 5.5. Hypotheses (H2b) and (H3b) ensure $\lambda$-uniformity where necessary.

Proposition 6.2. If ( $\mathrm{H} 1)$ holds, then $(\mathrm{H} 2 \mathrm{~b})$ is equivalent to $(\mathrm{H} 2)$ to hold for all $\lambda \in[a, b]$.

Proof. Assume that for all $\lambda \in[a, b]$ there exists $n(\lambda)$ and $C_{\lambda}$ such that for all $h \leq h(\lambda)$ and $\underline{z}_{h} \in X_{h}$

$$
\left\|\underline{z}_{n}\right\|_{X} \leq C_{\lambda}\left\|A_{h, \lambda} \underline{z}_{h}\right\|_{Y}
$$

Then, by hypothesis (H1), for all $\mu, \lambda$ we have

$$
\left\|\underline{z}_{h}\right\|_{X} \leq C_{\lambda}\left(C\left\|\left(\mathrm{D} G\left(\underline{x}_{\lambda}, \lambda\right)-\mathrm{D} G\left(\underline{x}_{\mu}, \mu\right)\right) \underline{z}_{h}\right\|_{Y}+\left\|A_{n, \mu} \underline{z}_{h}\right\|_{Y}\right) \text {. }
$$

Take $f(\mu):=\mathrm{D} G\left(\underline{x}_{\mu}, \mu\right)$. By the regularity of $\mathrm{D} G$ and the smoothness of the solutions with respect to the parameter it follows that $f \in \mathrm{C}^{\infty}([a, b] ; \mathcal{L}(X ; Y))$. Let $K^{\prime}:=C \max _{a \leq \mu \leq b}\left\|f^{\prime}(\mu)\right\|$. Then

$$
\left\|\underline{z}_{h}\right\|_{X} \leq C_{\lambda}\left(K^{\prime}|\lambda-\mu|\left\|\underline{z}_{h}\right\|_{X}+\left\|A_{h, \mu} \underline{z}_{h}\right\|_{Y}\right)
$$

Take $\varepsilon(\lambda)>0$ such that $\varepsilon(\lambda) C_{\lambda} K^{\prime} \leq 1 / 2$ and $I_{\lambda}:=[\max (a, \lambda-\varepsilon(\lambda)), \min (b, \lambda+\varepsilon(\lambda))]$. Then for all $h \leq h(\lambda)$ and $\mu \in I_{\lambda}$ we have

$$
\left\|\underline{z}_{h}\right\|_{X} \leq 2 C_{\lambda}\left\|A_{h, \mu} \underline{z}_{h}\right\|_{Y}
$$

We then take $\lambda_{1}, \ldots, \lambda_{N}$ such that $I_{\lambda_{j}}$ cover $[a, b]$ and $h_{0}:=\min h\left(\lambda_{j}\right)$ and finish there with the proof of the result.

By Proposition 6.2, we do not have to take care of uniformity in Hypothesis (H2). Therefore to have uniformity we just have to find uniform bounds on the convergence $\widetilde{P}_{h} \underline{x}_{\lambda} \rightarrow \underline{x}_{\lambda}$. This will be straightforward in all the methods we analyse.

Notice that these results justify the use of a continuation method in the regular branch for the numerical approximation using different values of the parameter $\lambda$. 


\section{Discretized multiplication operators}

Before studying the trigonometric approximations we recall some important tools which can be found with more details in $[11,12,15]$.

Let $a$ be a $\mathrm{C}^{\infty} 2 \pi$-periodic function. We denote by $\boldsymbol{a}$ the multiplication operator $\boldsymbol{a}: v \mapsto a v$. The following commutator property plays a fundamental role in our analysis (see (8) for the definition of $P$ and $Q$ )

the operator $P \boldsymbol{a}-\boldsymbol{a} P=\boldsymbol{a} Q-Q \boldsymbol{a}$ is compact from $\mathrm{H}^{s}$ into $\mathrm{H}^{s}$.

Indeed we deduce from (9) that we have

$$
P(a v)-a(P v)=\int_{0}^{2 \pi} k(\cdot, \theta) v(\theta) \mathrm{d} \theta, \quad \text { with } k(t, \theta):=\frac{e^{\imath \theta}}{2 \pi} \frac{a(t)-a(\theta)}{e^{\imath t}-e^{\imath \theta}}
$$

The function $k$ is clearly $\mathrm{C}^{\infty}$, which implies the compactness of the corresponding integral operator.

We introduce some notations

$$
\begin{gathered}
\mathrm{H}_{+}^{s}:=P \mathrm{H}^{s}, \quad \mathrm{H}_{-}^{s}:=Q \mathrm{H}^{s}, \quad \mathbb{T}_{n}^{+}:=P \mathbb{T}_{n}, \quad \mathbb{T}_{n}^{-}:=Q \mathbb{T}_{n}, \\
\pi_{n}^{+}:=P \pi_{n}=\pi_{n} P, \quad \pi_{n}^{-}:=Q \pi_{n}=\pi_{n} Q .
\end{gathered}
$$

We assume furthermore that the $2 \pi$-periodic function $a$ satisfies

$$
a \in \mathrm{C}^{\infty}, \quad a(t) \neq 0, \quad \forall t, \quad \operatorname{ind}(a)=0 .
$$

This implies that we can find a $2 \pi$-periodic $\mathrm{C}^{\infty}$ determination of the complex $\log \operatorname{arithm} \log a$. Then we set

$$
a_{+}:=\exp (P \log a), \quad a_{-}:=\exp (Q \log a)
$$

(the definitions are independent of the choice of the determinations), so that we have

$$
a=a_{+} a_{-}, \quad a_{+} \in \mathrm{C}^{\infty} \cap \mathrm{H}_{+}^{s}, \quad a_{-} \in \mathrm{C}^{\infty} \cap\left(\mathrm{H}_{-}^{s} \oplus \mathbb{R}\right) .
$$

The following properties are then easy to verify

$$
\begin{array}{ll}
\pi_{n}^{+} \boldsymbol{a}_{+} \pi_{n}^{+}=\pi_{n}^{+} \boldsymbol{a}_{+} P, & \pi_{n}^{-} \boldsymbol{a}_{-} \pi_{n}^{-}=\pi_{n}^{-} \boldsymbol{a}_{-} Q, \\
\pi_{n}^{+} \boldsymbol{a}_{-} \pi_{n}^{+}=P \boldsymbol{a}_{-} \pi_{n}^{+}, & \pi_{n}^{-} \boldsymbol{a}_{+} \pi_{n}^{-}=Q \boldsymbol{a}_{-} \pi_{n}^{-} .
\end{array}
$$

In the following lemma $J$ is a nonfinite subset of $\mathbb{N}^{*}$. The limits will be considered as $n \rightarrow \infty$ with $n \in J$.

Lemma 7.1. Let $\varepsilon$ and $\sigma$ belong to $\{+,-\}$.

(a) If $\left\{v_{n}\right\}_{n \in J}$ is a sequence such that $v_{n} \in \mathbb{T}_{n}^{\varepsilon}$, we have

$$
v_{n} \rightarrow 0 \text { and } \pi_{n}^{\varepsilon} \boldsymbol{a}_{\sigma} v_{n} \rightarrow 0 \text { in } \mathrm{H}^{s} \quad \Longrightarrow \quad v_{n} \rightarrow 0 \text { in } \mathrm{H}^{s}
$$

(b) If $\left\{v_{n}\right\}_{n \in J}$ is a sequence such that $v_{n} \in \mathbb{T}_{n}$, we have

$$
v_{n} \rightarrow 0 \text { and } \pi_{n} \boldsymbol{a} v_{n} \rightarrow 0 \text { in } \mathrm{H}^{s} \quad \Longrightarrow \quad v_{n} \rightarrow 0 \text { in } \mathrm{H}^{s} \text {. }
$$


Proof. Ad (a). We only consider the case $\varepsilon=+, \sigma=-$, the other cases being similar. We assume thus that $\mathbb{T}_{n}^{+} \ni v_{n} \rightarrow 0$ and $\pi_{n}^{+} \boldsymbol{a}_{-} v_{n} \rightarrow 0$ in $\mathrm{H}^{s}$. We introduce $b_{-}:=1 / a_{-}=\exp (-Q \log a) \in \mathrm{C}^{\infty}$. Using (27), we have (note that $\pi_{n}^{+} v_{n}=v_{n}$ )

$$
\begin{aligned}
v_{n} & =\pi_{n}^{+} \boldsymbol{b}_{-} \boldsymbol{a}_{-} \pi_{n}^{+} v_{n}=\pi_{n}^{+} \boldsymbol{b}_{-} P \boldsymbol{a}_{-} \pi_{n}^{+} v_{n}+\pi_{n}^{+} \boldsymbol{b}_{-}\left(\boldsymbol{a}_{-} P-P \boldsymbol{a}_{-}\right) \pi_{n}^{+} v_{n} \\
& =\pi_{n}^{+} \boldsymbol{b}_{-}\left(\pi_{n}^{+} \boldsymbol{a}_{-} v_{n}+\left(\boldsymbol{a}_{-} P-P \boldsymbol{a}_{-}\right) v_{n}\right)
\end{aligned}
$$

but $\pi_{n}^{+}$and $\boldsymbol{b}_{-}$are bounded in $\mathrm{H}^{s}$ and by compactness $(25)\left(\boldsymbol{a}_{-} P-P \boldsymbol{a}_{-}\right) v_{n} \rightarrow 0$ in $\mathrm{H}^{s}$. Hence $v_{n} \rightarrow 0$ in $\mathrm{H}^{s}$.

Ad (b). We assume now that $v_{n} \in \mathbb{T}_{n}, v_{n} \rightarrow 0$ and $\pi_{n} \boldsymbol{a} v_{n} \rightarrow 0$ in $\mathrm{H}^{s}$. Consider the operator

$$
C:=(\boldsymbol{a} P-P \boldsymbol{a}) P+(\boldsymbol{a} Q-Q \boldsymbol{a}) Q-P \boldsymbol{a}_{+}\left(P \boldsymbol{a}_{-}-\boldsymbol{a}_{-} P\right) P-Q \boldsymbol{a}_{-}\left(Q \boldsymbol{a}_{+}-\boldsymbol{a}_{+} Q\right) Q .
$$

By (25), $C$ is a compact operator from $\mathrm{H}^{s}$ into $\mathrm{H}^{s}$. Using that $P^{2}=P, Q^{2}=Q$ and $P+Q=I d$, we obtain

$$
C=\boldsymbol{a}-P \boldsymbol{a}_{+} P \boldsymbol{a}_{-} P-Q \boldsymbol{a}_{-} Q \boldsymbol{a}_{+} Q
$$

Left and right multiplication of this relation by $\pi_{n}$ and (27) yield

$$
\pi_{n} C \pi_{n}=\pi_{n} \boldsymbol{a} \pi_{n}-\pi_{n}^{+} \boldsymbol{a}_{+} \pi_{n}^{+} \boldsymbol{a}_{-} \pi_{n}^{+}-\pi_{n}^{-} \boldsymbol{a}_{-} \pi_{n}^{-} \boldsymbol{a}_{+} \pi_{n}^{-} .
$$

Since $C$ is compact we deduce that $\pi_{n} C \pi_{n} v_{n}=\pi_{n} C v_{n} \rightarrow 0$ and by hypothesis $\pi_{n} \boldsymbol{a} v_{n} \rightarrow 0$. Therefore (note that $\mathrm{H}_{+}^{s}$ and $\mathrm{H}_{-}^{s}$ are orthogonal)

$$
\pi_{n}^{+} \boldsymbol{a}_{+}\left(\pi_{n}^{+} \boldsymbol{a}_{-} \pi_{n}^{+} v_{n}\right) \rightarrow 0 \quad \text { and } \quad \pi_{n}^{-} \boldsymbol{a}_{-}\left(\pi_{n}^{-} \boldsymbol{a}_{+} \pi_{n}^{-} v_{n}\right) \rightarrow 0 \quad \text { in } \mathrm{H}^{s} .
$$

Using part (a) of this lemma we deduce

$$
\pi_{n}^{+} \boldsymbol{a}_{-} \pi_{n}^{+} v_{n} \rightarrow 0 \quad \text { and } \quad \pi_{n}^{-} \boldsymbol{a}_{+} \pi_{n}^{-} v_{n} \rightarrow 0 \quad \text { in } \mathrm{H}^{s}
$$

By using (a) again we have that $\pi_{n}^{+} v_{n} \rightarrow 0$ and $\pi_{n}^{-} v_{n} \rightarrow 0$ in $\mathrm{H}^{s}$, from where $v_{n}=\left(\pi_{n}^{+}+\pi_{n}^{-}\right) v_{n} \rightarrow 0$ in $\mathrm{H}^{s}$.

It is then easy to deduce from Lemma 7.1 (follow the proof in Lem. 5.6) the following proposition which means that the linear operator $\pi_{n} \boldsymbol{a}$ is uniformly invertible in $\mathbb{T}_{n}$ for $n$ great enough.

Proposition 7.2. Let $s$ be a nonnegative real number and a be a $2 \pi$-periodic function satisfying (25). Then there exist an integer $n_{0}$ and a real number $C>0$ such that

$$
\forall n \geq n_{0}, \forall v_{n} \in \mathbb{T}_{n}, \quad\left\|\pi_{n} \boldsymbol{a} v_{n}\right\|_{s} \geq C\left\|v_{n}\right\|_{s} .
$$

\section{Trigonometric Galerkin}

The trigonometric Galerkin method (sometimes referred as reduction method) can be written in the standard form (18) by means of the operator $P_{h}:=\left(\pi_{n}, \pi_{n}, 1\right)$ with $Y_{h}:=\mathbb{T}_{n}^{\mathbb{R}} \times \mathbb{T}_{n}^{\mathbb{R}} \times \mathbb{R}$, being $\mathbb{T}_{n}^{\mathbb{R}}:=\mathbb{T}_{n} \cap \mathrm{H}_{\mathbb{R}}^{0}$ the space of real valued trigonometric polynomials of degree at most $n$. Obviously $P_{h}$ satisfies (H1).

Consider the operator $\widetilde{P}_{h}: X \rightarrow x_{0} \mathbb{T}_{n} \times \mathbb{R}=: X_{h} \subset X$,

$$
\widetilde{P}_{h}(x, c):=\left(x_{0} \pi_{n}\left(\bar{x}_{0} x\right), c\right) .
$$

We remark that for $g \in \mathrm{H}^{t}$ and $s \leq t$,

$$
\left\|g-x_{0} \pi_{n}\left(\bar{x}_{0} g\right)\right\|_{s} \leq C n^{s-t}\|g\|_{t} .
$$

Since the solution of (17) is smooth, then Hypotheses (H3) and (H3b) hold. Therefore we are left to proving the $h$-uniform invertibility of $A_{h}:=\left.P_{h} \mathrm{D} G(\underline{x})\right|_{X_{h}}: X_{h} \rightarrow Y_{h}($ recall that $h=1 / n)$. 
Theorem 8.1. There exist $h_{0}>0$ and $C>0$ such that for all $\underline{z}_{h} \in X_{h}$ and $h \leq h_{0}$,

$$
\left\|\underline{z}_{h}\right\|_{X} \leq C\left\|A_{h} \underline{z}_{h}\right\|_{Y} .
$$

Proof. We prove this result by means of Lemma 5.6. Notice first that we have $\left\|P_{h} y-y\right\|_{Z} \leq C n^{-1}\|y\|_{Y}$ with $Z:=\mathrm{H}_{\mathbb{R}}^{1} \times \mathrm{H}_{\mathbb{R}}^{0} \times \mathbb{R}$, and thus the Hypothesis (a) holds (see Rem. 5.7). Let then $y_{n} \in x_{0} \mathbb{T}_{n}$ be such that

$$
y_{n} \rightarrow 0, \quad \text { in } \mathrm{H}^{2}, \quad P_{h} A_{0}\left(a y_{n}, 0\right) \rightarrow 0 \quad \text { in } Y,
$$

with $a$ given in Proposition 4.3. The sequences $y_{n}$ and $u_{n}:=\bar{x}_{0} y_{n} \in \mathbb{T}_{n}$ converge to zero weakly in $\mathrm{H}^{2}$ and therefore strongly in $\mathrm{C}^{0}$. Since $\pi_{n}$ commutes with the operators $\operatorname{Re}$, Im and $Q$ we deduce from (12) that

$$
P_{h} A_{0}\left(a y_{n}, 0\right)-A_{0}\left(x_{0} \pi_{n} \boldsymbol{a} u_{n}, 0\right)=\left(0,0, \delta_{n}\right)
$$

with $\delta_{n} \rightarrow 0$. From (29) and since $A_{0}$ is an isomorphism, it follows readily that $\pi_{n} \boldsymbol{a} u_{n} \rightarrow 0$ in $\mathrm{H}^{2}$ and using Proposition 7.2, $u_{n} \rightarrow 0$ in $\mathrm{H}^{2}$ and hence so does $y_{n}$. Thus, Lemma 5.6 proves the result.

By Theorem 5.5 and the definition of $\widetilde{P}_{h}$ we obtain

$$
\left|c_{h}\right|+\left\|x_{h}-x\right\|_{2} \leq\left\|\underline{x}_{h}-\underline{x}\right\|_{X} \leq C\left\|\pi_{n}\left(\bar{x}_{0} x\right)-\bar{x}_{0} x\right\|_{2} .
$$

Since $x$ is analytic, as an easy consequence of Theorem 11.5 in [10] it follows that for some $\sigma>0$

$$
\left|c_{h}\right|+\left\|x_{h}-x\right\|_{2} \leq C e^{-\sigma n} .
$$

\section{TRIgONOMETRIC COLLOCATION}

Let $\widetilde{P}_{h}$ be as in the preceding section. Consider the interpolation operators

$$
Q_{n}^{j} f \in \mathbb{T}_{n}, \quad Q_{n}^{j} f\left(\theta_{k}^{j}\right)=f\left(\theta_{k}^{j}\right), \quad \forall k
$$

for $j=1,2$. By an elementary uniqueness argument it follows that if $f$ is real valued, so is $Q_{n}^{j} f$. Consider also the operator $P_{h}: Y \rightarrow Y_{h}:=\mathbb{T}_{n}^{\mathbb{R}} \times \mathbb{T}_{n}^{\mathbb{R}} \times \mathbb{R}$,

$$
P_{h}(f, g, c):=\left(Q_{n}^{1} f, Q_{n}^{2} g, c\right) .
$$

With these new notations, it is obvious that (19) is equivalent to (18). If $Q_{n}$ is any of $Q_{n}^{j}$ and $t>1 / 2$, then for all $s \in[0, t]$ there exists a constant $C$ such that for all $f \in \mathrm{H}^{t}$

$$
\left\|Q_{n} f-f\right\|_{s} \leq C n^{s-t}\|f\|_{t} .
$$

The proof of this result can be found in [16] Lemma 2.1. The uniform boundedness of $P_{h}$, that is, Hypothesis (H1), is a direct consequence of (31).

Theorem 9.1. There exist $h_{0}>0$ and $C>0$ such that for all $\underline{z}_{h} \in X_{h}$ and $h \leq h_{0}$,

$$
\left\|\underline{z}_{h}\right\|_{X} \leq C\left\|A_{h} \underline{z}_{h}\right\|_{Y} .
$$

Proof. We apply again Lemma 5.6. By (31) it follows readily that $\left\|P_{h} y-y\right\|_{Z} \leq C n^{-1}\|y\|_{Y}$ with $Z:=$ $\mathrm{H}_{\mathbb{R}}^{1} \times \mathrm{H}_{\mathbb{R}}^{0} \times \mathbb{R}$ and thus (a) holds. Thus, let $y_{n} \in x_{0} \mathbb{T}_{n}$ satisfy (29).

The operator $Q \boldsymbol{a}-\boldsymbol{a} Q$ is compact in $\mathrm{H}^{2}$ (see (25)). Therefore (29) implies that

$$
Q_{n}^{1} \operatorname{Re}\left(a Q\left(\bar{x}_{0} y_{n}\right)\right) \rightarrow 0, \quad \text { in } \mathrm{H}^{2} .
$$


Since $z_{n}^{-}:=Q\left(\bar{x}_{0} y_{n}\right) \in \mathbb{T}_{n}^{-}=Q \mathbb{T}_{n}$ satisfies $z_{n}^{-} \rightarrow 0$ in $\mathrm{H}^{2}$, by Lemma 9.2 it follows that

$$
z_{n}^{-} \rightarrow 0, \quad \text { in } \mathrm{H}^{2}
$$

By the compact inclusion of $\mathrm{H}^{2}$ into $\mathrm{H}^{1}$ and the first convergence in (29), it follows that $y_{n} \rightarrow 0$ in $\mathrm{H}^{1}$. Let now $z_{n}:=\bar{x}_{0} y_{n}$ and $z_{n}^{+}:=z_{n}-z_{n}^{-}$. The result will be proven once we see that $z_{n}^{+} \rightarrow 0$ in $\mathrm{H}^{2}$.

To see that, notice first that

$$
\begin{aligned}
\operatorname{Im} Q_{n}^{2}\left(a z_{n}^{\prime}\right) & =\operatorname{Im} Q_{n}^{2}\left(\left(a z_{n}\right)^{\prime}-a^{\prime} z_{n}\right) \\
& =\operatorname{Im} Q_{n}^{2}\left(\bar{x}_{0}\left(a y_{n}\right)^{\prime}\right)+\operatorname{Im} Q_{n}^{2}\left(\left(a \bar{x}_{0}^{\prime}-a^{\prime} \bar{x}_{0}\right) y_{n}\right)
\end{aligned}
$$

By (29) and the fact that $y_{n} \rightarrow 0$ in $\mathrm{H}^{1}$, it follows that the right-hand side of (34) converges to zero in $\mathrm{H}^{1}$. We define now $v_{n}:=\left(z_{n}^{+}\right)^{\prime}$ and remark that $\bar{v}_{n} \in \mathbb{T}_{n}^{-}$. By (33) it follows that

$$
-\operatorname{Re}\left(Q_{n}^{2}\left(\imath \bar{a} \bar{v}_{n}\right)\right)=\operatorname{Im} Q_{n}^{2}\left(a v_{n}\right) \rightarrow 0, \quad \text { in } \mathrm{H}^{1} .
$$

Moreover, $\bar{v}_{n} \rightarrow 0$ in $\mathrm{H}^{1}$. Hence, Lemma 9.2 proves that $v_{n} \rightarrow 0$ in $\mathrm{H}^{1}$, and therefore, $z_{n}^{+} \rightarrow 0$ in $\mathrm{H}^{2}$.

Lemma 9.2. Let $s>1 / 2, a \in \mathrm{C}^{\infty}$ be such that $a(t) \neq 0$ for all $t$ and $Q_{n}$ be any of $Q_{n}^{j}$. Let $u_{n} \in \mathbb{T}_{n}^{-}$for $n \geq 1$ be such that $u_{n} \rightarrow 0$ in $\mathrm{H}^{s}$ and that $\operatorname{Re} Q_{n}\left(a u_{n}\right) \rightarrow 0$ in $\mathrm{H}^{s}$. Then $u_{n}$ converges strongly to zero in $\mathrm{H}^{s}$.

Proof. All convergences in this proof are considered in the space $\mathrm{H}^{s}$ without explicit indication. Let $d:=\bar{a} / a$ and consider the sequence

$$
w_{n}:=\frac{1}{2} u_{n}+\frac{1}{2} Q_{n}\left(d \bar{u}_{n}\right)
$$

which satisfies

$$
Q_{n}\left(a w_{n}\right)=\frac{1}{2} Q_{n}\left(a u_{n}\right)+\frac{1}{2} Q_{n}\left(a Q_{n}\left((\bar{a} / a) \bar{u}_{n}\right)\right)=\operatorname{Re} Q_{n}\left(a u_{n}\right) \rightarrow 0
$$

Hence

$$
w_{n}=Q_{n}\left(\frac{1}{a} Q_{n}\left(a w_{n}\right)\right) \rightarrow 0 .
$$

Let $d_{n}:=\pi_{n} d$. Since $d_{n}-d \rightarrow 0,(35)$ and the uniform boundedness of $u_{n}$ imply that

$$
u_{n}+Q_{n}\left(d_{n} \bar{u}_{n}\right) \rightarrow 0
$$

Since $P u_{n}=0,(36)$ implies $P Q_{n}\left(d_{n} \bar{u}_{n}\right) \rightarrow 0$. On the other hand (recall the definitions of $\pi_{n}^{+}$and $\pi_{n}^{-}$in $(26))$

$$
d_{n} \bar{u}_{n}=\pi_{n}^{+}\left(d_{n} \bar{u}_{n}\right)+\pi_{n}^{-}\left(d_{n} \bar{u}_{n}\right)+\left(\pi_{2 n}^{+}-\pi_{n}^{+}\right)\left(d_{n} \bar{u}_{n}\right)
$$

and therefore

$$
Q_{n}\left(d_{n} \bar{u}_{n}\right)=\pi_{n}^{+}\left(d_{n} \bar{u}_{n}\right)+\pi_{n}^{-}\left(d_{n} \bar{u}_{n}\right)+Q_{n}\left(\pi_{2 n}^{+}-\pi_{n}^{+}\right)\left(d_{n} \bar{u}_{n}\right)
$$

Since $\left(\pi_{2 n}^{+}-\pi_{n}^{+}\right)\left(d_{n} \bar{u}_{n}\right) \in \operatorname{span}\left(\phi_{n+1}, \ldots, \phi_{2 n}\right)$, it follows that $Q_{n}\left(\pi_{2 n}^{+}-\pi_{n}^{+}\right)\left(d_{n} \bar{u}_{n}\right) \in \mathbb{T}_{n}^{-}$and thus

$$
\pi_{n}^{+}\left(d_{n} \bar{u}_{n}\right)=P Q_{n}\left(d_{n} \bar{u}_{n}\right)=P\left(u_{n}+Q_{n}\left(d_{n} \bar{u}_{n}\right)\right) \rightarrow 0 .
$$


Applying again the strong convergence of $d_{n}$ towards $d$ and the boundedness of the sequence $u_{n}$, (37) gives $\pi_{n}^{+}\left(d \bar{u}_{n}\right) \rightarrow 0$.

Since $\bar{u}_{n}=P \bar{u}_{n}$, we have $\pi_{n} \boldsymbol{d} \bar{u}_{n}=\pi_{n}^{+} \boldsymbol{d} \bar{u}_{n}+\pi_{n}(\boldsymbol{d} P-P \boldsymbol{d}) u_{n}$. Using the assumption $u_{n} \rightarrow 0$ and the compactness of $\boldsymbol{d} P-P \boldsymbol{d}$ (see (25)) we obtain that $\pi_{n} \boldsymbol{d} \bar{u}_{n} \rightarrow 0$. Then $u_{n} \rightarrow 0$ follows from Proposition 7.2.

Remark 9.3. This lemma is valid for any non vanishing smooth function $a$.

Obviously the last remarks of the previous Section hold in this new situation and (30) is valid for the trigonometric collocation method.

\section{Spline Galerkin method}

Let $\Delta_{h}=\left\{t_{j}: j \in \mathbb{Z}\right\}$ be a partition of $\mathbb{R}$ satisfying the conditions given in Section 5 and $h:=\max \left|t_{j+1}-t_{j}\right|$. We consider the discrete spaces $X_{h}:=S^{d}\left(\Delta_{h}\right) \times \mathbb{R}$ and $Y_{h}:=S_{\mathbb{R}}^{d}\left(\Delta_{h}\right) \times S_{\mathbb{R}}^{d-1}\left(\Delta_{h}\right) \times \mathbb{R}$ for $d \geq 2$. Let $\widetilde{P}_{h}: X \rightarrow X_{h}$ be given by

$$
\widetilde{P}_{h}(f, c):=\left(P_{h, 2}^{d} f, c\right)
$$

being $P_{h, 2}^{d}$ the $\mathrm{H}^{2}$-orthogonal projection onto $S^{d}\left(\Delta_{h}\right)$. It is well-known (see [15], Th. 2.7) that for all $g \in \mathrm{H}^{d+1}$

$$
\left\|g-P_{h, 2}^{d} g\right\|_{2} \leq C h^{d-1}\|g\|_{d+1} .
$$

Therefore, Hypotheses (H3) and (H3b) hold.

By $P_{h}^{d}: \mathrm{H}^{0} \rightarrow S^{d}\left(\Delta_{h}\right)$ we denote the $\mathrm{H}^{0}$-orthogonal projections onto $S^{d}\left(\Delta_{h}\right)$. We remark that these operators respect real and imaginary parts. If we define the projections $P_{h}: Y \rightarrow Y_{h}$ by

$$
P_{h}(f, g, c):=\left(P_{h}^{d} f, P_{h}^{d-1} g, c\right),
$$

the spline Galerkin method is equivalent to (18).

Hypothesis $\left(\mathrm{G}_{d}\right)$. There exist $C_{1}, C_{2}, C_{3}>0$, independent of $h$ such that for all $h$ small enough:

(a) $\left\|P_{h}^{d} g\right\|_{2} \leq C_{1}\|g\|_{2}$ for all $g \in \mathrm{H}^{2}$;

(b) $\left\|P_{h}^{d-1} g\right\|_{1} \leq C_{2}\|g\|_{1}$ for all $g \in \mathrm{H}^{1}$;

(c) for all $u_{h} \in S^{d}\left(\Delta_{h}\right)$ and $f \in \mathrm{C}^{\infty}$

$$
\left\|f u_{h}-P_{h}^{d}\left(f u_{h}\right)\right\|_{2} \leq C_{3} h\left\|u_{h}\right\|_{2} .
$$

Obviously, (a) and (b) mean that (H1) holds. Using again Theorem 2.7 of [15] we have

$$
\left\|g-P_{h}^{d} g\right\|_{0}+\left\|g-P_{h}^{d-1} g\right\|_{0} \leq C h\|g\|_{1}, \quad \forall g \in \mathrm{H}^{1} .
$$

Therefore, with $Z:=\mathrm{H}_{\mathbb{R}}^{0} \times \mathrm{H}_{\mathbb{R}}^{0} \times \mathbb{R}$ we have $\left\|y-P_{h} y\right\|_{Z} \leq C h\|y\|_{Y}$ and the assumption (a) of Lemma 5.6 holds.

Proposition 10.1. If the sequence of grids is quasi-uniform, that is, if there exists $\lambda>0$ such that

$$
\min _{1 \leq i \leq n}\left|t_{i+1}-t_{i}\right| \geq \lambda h,
$$

then hypothesis $\left(\mathrm{G}_{d}\right)$ is satisfied for all $d \geq 2$.

Proof. Parts (a) and (b) follows readily by a straightforward application of the inverse inequalities and convergence properties of $P_{h}^{d}$ in $\mathrm{H}^{0}$ (see [15] Ths. 2.11 and 2.7). Then, with the aid of (a), Theorem 2.13 of [15] proves (c). 
Theorem 10.2. If the sequence $\left\{\Delta_{h}\right\}$ satisfies $\left(\mathrm{G}_{d}\right)$, then there exist $h_{0}>0$ and $C>0$ such that for all $\underline{z}_{h} \in X_{h}$ and $h \leq h_{0}$,

$$
\left\|\underline{z}_{h}\right\|_{X} \leq C\left\|A_{h} \underline{z}_{h}\right\|_{Y}
$$

Proof. Proceeding as usual, we consider a sequence $y_{h} \in S^{d}\left(\Delta_{h}\right)$ such that

$$
y_{h} \rightarrow 0, \quad \text { in } \mathrm{H}^{2}, \quad \operatorname{Re} P_{h}^{d}\left(Q\left(a \bar{x}_{0} y_{h}\right)\right) \rightarrow 0, \quad \text { in } \mathrm{H}^{2}, \quad \operatorname{Im} P_{h}^{d-1}\left(\left(a \bar{x}_{0} y_{h}\right)^{\prime}\right) \rightarrow 0, \quad \text { in } \mathrm{H}^{1} .
$$

Let $u_{h}:=P_{h}^{d}\left(a \bar{x}_{0} y_{h}\right) \in S^{d}\left(\Delta_{h}\right)$. By part (c) of Hypothesis $\left(\mathrm{G}_{d}\right)$, it follows that

$$
\left\|u_{h}-a \bar{x}_{0} y_{h}\right\|_{2} \leq C h\left\|y_{h}\right\|_{2} \leq C^{\prime} h \rightarrow 0
$$

and hence $u_{h} \rightarrow 0$ in $\mathrm{H}^{2}$. From these results we can easily arrive at the following convergences:

$$
\hat{u}_{h}(0) \rightarrow 0, \quad \operatorname{Re} P_{h}^{d}\left(Q u_{h}\right) \rightarrow 0, \quad \text { in } \mathrm{H}^{2}, \quad \operatorname{Im} P_{h}^{d-1}\left(u_{h}^{\prime}\right) \rightarrow 0, \quad \text { in } \mathrm{H}^{1} .
$$

Decomposing $u_{h}$ into real and imaginary parts $u_{h}=v_{h}+\imath w_{h}$, we have

$$
w_{h}^{\prime}=P_{h}^{d-1}\left(w_{h}^{\prime}\right)=\operatorname{Im} P_{h}^{d-1}\left(u_{h}^{\prime}\right),
$$

since $w_{h}^{\prime} \in S_{\mathbb{R}}^{d-1}\left(\Delta_{h}\right)$. Hence by (41) we prove

$$
w_{h} \rightarrow 0, \quad \text { in } \mathrm{H}^{2} .
$$

We remark that if $v \in \mathrm{H}_{\mathbb{R}}^{0}$, then $2 \operatorname{Re} Q v=Q v+\overline{Q v}=v-\hat{v}(0)$. Consequently, by (41) and (42), it follows that

$$
v_{h}-\hat{v}_{h}(0)=P_{h}^{d}\left(v_{h}-\hat{v}_{h}(0)\right)=2 \operatorname{Re} P_{h}^{d}\left(Q v_{h}\right) \rightarrow 0, \quad \text { in } \mathrm{H}^{2} .
$$

This fact, together with (42) and (41) proves that $u_{h} \rightarrow 0$ in $\mathrm{H}^{2}$ and by (40), $y_{h} \rightarrow 0$ in $\mathrm{H}^{2}$.

Applying now Theorem 5.5, the definition of $\widetilde{P}_{h}$ and (38) we obtain

$$
\left|c_{h}\right|+\left\|x_{h}-x\right\|_{2} \leq\left\|\underline{x}_{h}-\underline{x}\right\|_{X} \leq C\left\|P_{h, 2}^{d} x-x\right\|_{2} \leq C h^{d-1}\|x\|_{d+1} .
$$

\section{Spline COLlocation}

Let $\left\{\Delta_{h}\right\}$ be a sequence of partitions such that the diameter $h$ converges to zero. If $d$ is even we consider the points

$$
\theta_{k}:=\frac{t_{k}+t_{k+1}}{2}, \quad k \in \mathbb{Z}
$$

whereas if $d$ is odd we consider

$$
\theta_{k}:=t_{k}, \quad k \in \mathbb{Z}
$$

Then we can define the spline interpolation operator onto $S^{d}\left(\Delta_{h}\right)$

$$
I_{h}^{d} g \in S^{d}\left(\Delta_{h}\right), \quad I_{h}^{d} g\left(\theta_{k}\right)=g\left(\theta_{k}\right), \quad \forall k .
$$

We remark that if $g$ is real valued, $I_{h}^{d} g$ is also real valued. We assume that the sequence of grids is such that the following hypothesis holds. 
Hypothesis $\left(\mathrm{C}_{d}\right)$. There exist $C_{1}, C_{2}, C_{3}>0$, independent of $h$ such that for $h$ small enough:

(a) $\left\|I_{h}^{d} g\right\|_{2} \leq C_{1}\|g\|_{2}$ for all $g \in \mathrm{H}^{2}$;

(b) $\left\|I_{h}^{d-1} g\right\|_{1} \leq C_{2}\|g\|_{1}$ for all $g \in \mathrm{H}^{1}$;

(c) for all $u_{h} \in S^{d}\left(\Delta_{h}\right)$ and $f \in \mathrm{C}^{\infty}$

$$
\left\|f u_{h}-I_{h}^{d}\left(f u_{h}\right)\right\|_{2} \leq C_{3} h\left\|u_{h}\right\|_{2} .
$$

Defining $X_{h}, Y_{h}$ and $\tilde{P}_{h}$ as in the previous section and $P_{h}: Y \rightarrow Y_{h}$ by

$$
P_{h}(f, g, c):=\left(I_{h}^{d} f, I_{h}^{d-1} g, c\right)
$$

the spline collocation method is obviously equivalent to (18). Besides, Hypothesis $\left(\mathrm{C}_{d}\right)$ assures that conditions (H1), (H3) and (H3b) hold.

Moreover, similarly to the Galerkin discretization we can deduce the estimates

$$
\left\|g-I_{h}^{d} g\right\|_{0} \leq C h\|g\|_{2}, \quad\left\|g-I_{h}^{d-1} g\right\|_{0} \leq C h\|g\|_{1} .
$$

For instance, to prove the first inequality let us consider $r_{h} g$ the piecewise linear continuous interpolate at the same points as $I_{h}^{d} g$. It is easy to see that $\left\|g-r_{h} g\right\|_{0} \leq C h\|g\|_{1}$ and, by the Poincaré inequality applied in each subinterval, that $\left\|I_{h}^{d} g-r_{h} g\right\|_{0} \leq C h\left\|I_{h}^{d} g-r_{h} g\right\|_{1}$. Then the result follows by the assumed stability of $I_{h}^{d}$. Therefore (44) implies that assumption (a) of Lemma 5.6 holds.

Proposition 11.1. If all the partitions are uniform, Hypothesis $\left(\mathrm{C}_{d}\right)$ is satisfied for all $d \geq 2$.

Proof. It follows from the interpolation theory for periodic splines on uniform grids together with the commutator property of splines (Ths. 2.30 and 2.13 of [15]).

Theorem 11.2. If the sequence $\left\{\Delta_{h}\right\}$ satisfies $\left(\mathrm{C}_{d}\right)$, then there exist $h_{0}$ and $C>0$ such that for all $\underline{z}_{h} \in X_{h}$ and $h \leq h_{0}$,

$$
\left\|\underline{z}_{h}\right\|_{X} \leq C\left\|A_{h} \underline{z}_{h}\right\|_{Y}
$$

Proof. Changing projections $P_{h}^{\alpha}(\alpha \in\{d, d-1\})$ by $I_{h}^{\alpha}$ in all their occurrences, the proof of Theorem 10.2 is valid.

Applying now Theorem 5.5, we obtain

$$
\left|c_{h}\right|+\left\|x_{h}-x\right\|_{2} \leq C h^{d-1}\|x\|_{d+1} .
$$

\section{Numerical TESTS}

We have tested the previous methods on the simple example where $j$ is the sum of two symmetric Dirac masses $j=\pi\left(\delta_{-1}+\delta_{+1}\right)$. In this case the free boundary is known [8] and described by the parametric equation

$$
z(\varphi)=\frac{1-a^{4}}{4 a \lambda}\left(\frac{2}{a e^{i \varphi}-a^{-1} e^{-i \varphi}}+\log \frac{1-a e^{i \varphi}}{1+a e^{i \varphi}}\right)
$$

where $a \in(0,1)$ is solution of

$$
4 \lambda=2 a+\frac{1-a^{4}}{a} \log \frac{1+a^{2}}{1-a^{2}}
$$


We have represented in Figure 1 the solutions corresponding to the values $a=0.4,0.6,0.8,0.99$ and in Figure 2, $\lambda$ as a function of $a$. We note that we have a limit value $\lambda=0.68273968 \ldots$. For $a=1, \lambda=0.5$ we lose connectedness and $\Omega$ become the union of two tangent circles.

For our numerical experiments we have used a continuation method for computing the solution, starting from $\lambda=0$. In order to avoid the singular situation related to the turning point we have used $t=\operatorname{Im}(x(\pi / 2)-$ $x(3 \pi / 2)$ as continuation parameter. The numerical behaviour confirms the theorical estimates: exponential (resp. polynomial) decay of the error in the trigonometric (resp. spline) case. Therefore in regular situations as $a=0.4,0.6$, or 0.8 trigonometric approximations are far better than spline ones but become more and more unstable as $a$ brings closer to 1 . They cannot be used for $a=0.99$ while spline approximations (with appropriate mesh refinement) are still working for this value.

Some additional material on numerical tests for this problem can be found in [5].

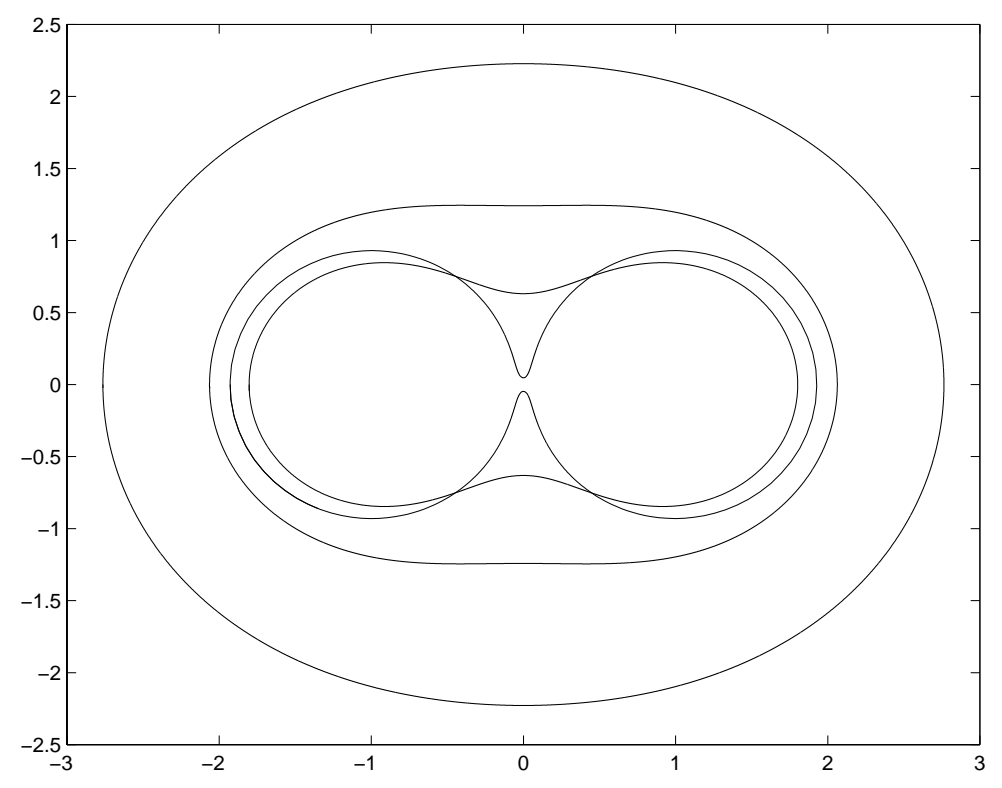

FiguRE 1. Solutions for $a=0.4,0.6,0.8,0.99$.

Acknowledgements. The third author is partially supported by DGES Project PB97-1013. Part of this work has been carried out during a stay of the third author at the Institut Mathématique de Rennes financed by the Université de Rennes I and during a stay of the first author at the Universidad de Zaragoza, supported by Programa Europa CAI.

\section{REFERENCES}

[1] H.W. Alt and L.A. Caffarelli, Existence and regularity for a minimum problem with a free boundary. J. Reine Angew. Math. 25 (1981) 105-144.

[2] O. Coulaud and A. Henrot, Numerical approximation of a free boundary problem arising in electromagnetic shaping. SIAM J. Numer. Anal. 31 (1994) 1109-1127.

[3] M. Crouzeix, Variational approach of magnetic shaping problem. Eur. J. Mech. B/Fluids 10 (1991) 627-536.

[4] J. Descloux, Stability of solutions of the bidimensional magnetic shaping problem in absence of surface tension. Eur. J. Mech. B/Fluids 10 (1991) 513-526.

[5] Ph. Féat, Approximation d'un problème de frontière libre bidimensionnel. Thèse de l'Université de Rennes I, France (1998).

[6] A. Friedman, Variational Principles and Free Boundary Problems. John Wiley \& Sons, New York (1982).

[7] B. Gustafsson and H. Shagholian, Existence and geometric properties of solutions of a free boundary problem in potential theory. J. Reine Angew. Math. 68 (1996) 137-179. 


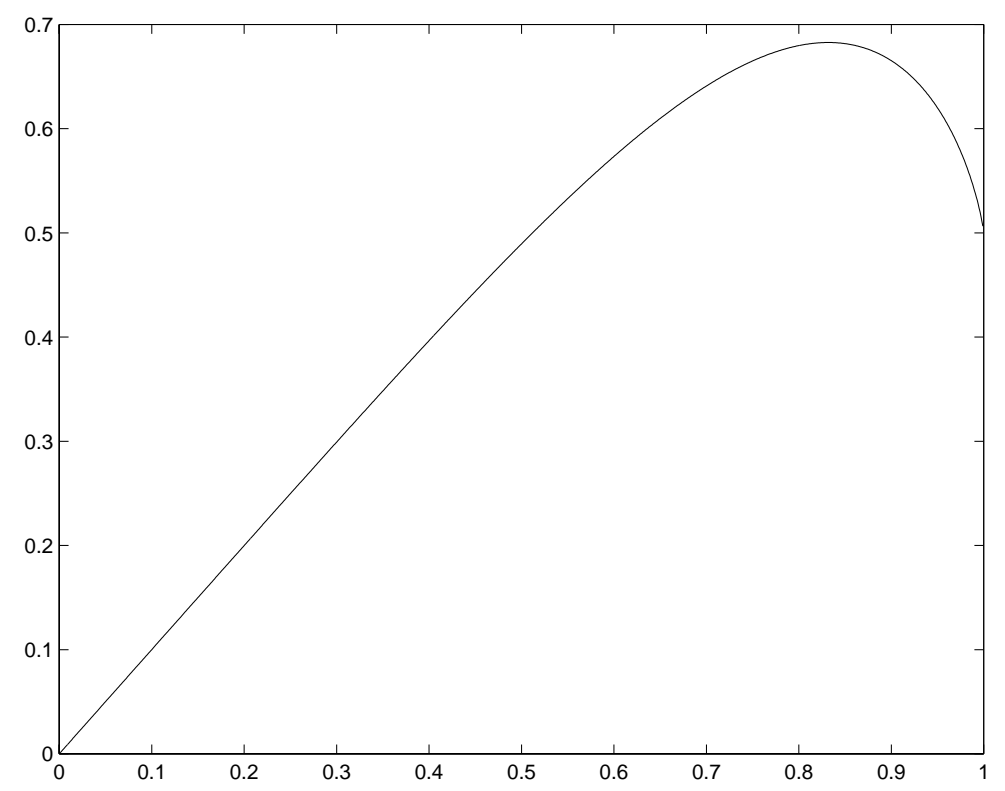

FIGURE 2. The parameter $\lambda$ as a function of the parameter $a$.

[8] A. Henrot, Subsolutions and supersolutions in a free boundary problem. Ark. Mat. 32 (1994) 79-98.

[9] A. Henrot and M. Pierre, Un problème inverse en formage des métaux liquides. RAIRO Modél. Math. Anal. Numér. 23 (1989) $155-177$.

[10] R. Kress, Linear Integral Equations. Springer, New York (1989).

[11] W. McLean and W.L. Wendland, Trigonometric approximation of solutions of periodic pseudodifferential equations. Oper. Theory: Adv. Appl. 41 (1989) 359-383.

[12] S. Mikhlin and S. Prößdorf, Singular Integral Operators. Springer-Verlag, Berlin (1986).

[13] X. Pelgrin, Un problème de frontière libre. Thèse de l'Université de Rennes I, France (1994).

[14] M. Pierre and J.R. Roche, Numerical simulation of tridimensional electromagnetic shaping of liquid metals. Numer. Math. 65 (1993) 203-217.

[15] S. Prößdorf and B. Silbermann, Numerical Analysis for Integral and Related Operator Equations. Akademie-Verlag, Berlin (1991).

[16] J. Saranen and L. Schroderus, Quadrature methods for strongly elliptic equations of negative order on smooth closed curves. SIAM J. Numer. Anal. 30 (1993) 1769-1795.

[17] Y. Yan and I.H. Sloan, On integral equations of the first kind with logarithmic kernels. J. Integral Equations. Appl. 1 (1988) $549-579$.

To access this journal online: www.edpsciences.org 